\title{
Accuracy of Potential Evapotranspiration Models in Different Time Scales
}

\author{
Lucas Eduardo de Oliveira Aparecido ${ }^{1}$ (D), Kamila Cunha de Meneses $^{2}$ (D), \\ Guilherme Botega Torsoni ${ }^{1}$ (D), José Reinaldo da Silva Cabral de Moraes $^{1}$ (D), \\ Daniel Zimmermann Mesquita ${ }^{1}$ \\ ${ }^{I}$ Instituto Federal do Mato Grosso do Sul, Naviraí, MS, Brasil. \\ ${ }^{2}$ Faculdade de Ciências Agrárias e Veterinárias, Universidade Estadual Paulista, Jaboticabal, \\ SP, Brasil.
}

Recebido em: 31 de Março de 2019 - Aceito em: 24 de Agosto de 2019

\begin{abstract}
Resumo
Conhecer a precisão dos modelos de evapotranspiração potencial em diferentes condições agronômicas e climáticas é útil para a agricultura irrigada. Portanto, objetivou-se comparar 18 métodos de estimativa de ETP com o método de Penman-Monteith (FAO-56), em diferentes escalas de tempo para o Estado de Mato Grosso do Sul. Foram utilizadas séries históricas dos dados climáticos em escala diária entre 1983 a 2018 de 22 localidades no estado do Mato Grosso do Sul. Os modelos de estimativa de ETP testados foram: Benevidez-Lopez, Blaney-Criddle, Camargo, Hamon, Hargreaves, Hargreaves-Samani, Jensen-Haise, Jobson, Kharrufa, Linacre, Makkink, Penman, Priestley-Taylor, Radiation, Romanenko, Tanner-Pelton, Thornthwaite e Turc. Esses modelos foram comparados com Penman-Monteith em escalas diárias, semanais e mensais. A comparação entre os modelos de estimativa de ETP e o modelo de Pernman-Monteith foi realizada pelos índices estatísticos: acurácia (MAPE) e precisão $\left(\mathrm{R}^{2} \mathrm{aj}\right)$. Os métodos de estimativa demonstraram diferenças na eficiência em relação às escalas de tempo. Os melhores desempenhos dos modelos foram na escala diária. Para escala diária, os métodos de Priestley-Taylor, Hargreaves, Hamon e Makkink apresentam os melhores valores de acurácia e precisão para o Estado de Mato Grosso do Sul. Para escala semanal os melhores métodos são Hamon e Makkink, enquanto para escala mensal os melhores métodos para estimar a ETP são Makkink e Priestley-Taylor.
\end{abstract}

Palavras-chave: métodos de estimativa, acurácia, precisão, Penman-Monteith, evaporação da água.

\section{Acurácia de Modelos de Evapotranspiração Potencial em Diferentes Escalas de Tempo}

\begin{abstract}
Know the precision of potential evapotranspiration models in different agronomic and climatic conditions is useful for irrigated agriculture. Therefore, we aimed to compare 18 methods of estimation of ETP with the Penman-Monteith (FAO-56) method, at different time scales for the State of Mato Grosso do Sul. Time series of climatic data were used on a daily scale between 1983 and 2018 from 22 locations in the state of Mato Grosso do Sul. ETP estimation models tested were: Benevidez-Lopez, Blaney-Criddle, Camargo, Hamon, Hargreaves, Hargreaves-Samani, Jensen-Haise, Jobson, Kharrufa, Linacre, Makkink, Penman, Priestley-Taylor, Radiation, Romanenko, Tanner-Pelton, Thornthwaite, and Turc. These models were compared with Penman-Monteith in daily, weekly, and monthly scales. The comparison between the ETP estimation models and the Penman-Monteith model was performed by the statistical indices: accuracy (MAPE) and precision $\left(R^{2}\right.$ aj). Estimation methods showed differences in efficiency over time scales. The best performances of the models were on the daily scale. For daily scale, methods of Priestley-Taylor, Hargreaves, Hamon, and Makkink present the best values of accuracy and precision for the State of Mato Grosso do Sul. In the weekly scale, the most accurate methods are Hamon and Makkink, while for monthly scale the best methods are Makkink and Priestley-Taylor.
\end{abstract}

Keywords: estimation methods, accuracy, precision, Penman-Monteith, water evaporation.

Autor de correspondência: Lucas Eduardo de Oliveira Aparecido, lucas.aparecido@ifms.edu.br. 


\section{Introdução}

O Mato Grosso do Sul tem destaque no cenário agrícola brasileiro, principalmente na produção de soja, sorgo, milho e cana-de-açúcar. Esses cultivos dependem de irrigação suplementar para conseguir altas produtividades (Cunha et al., 2017a). A existência de veranicos tem limitado o potencial genético dessas culturas agrícolas neste estado. Uma forma de amenizar esses veranicos é utilizar da irrigação, mas sem desperdícios. O desperdício de água é a principal causa de sua escassez em diversos países em desenvolvimento (Tao et al., 2018).

A estimativa da evapotranspiração potencial (ETP) é fundamental no planejamento da irrigação, na avaliação da produtividade dos cultivos agrícolas e nas pesquisas ambientais, uma vez que a ETP é um componente essencial no ciclo hidrológico (Adamala, 2018; Almorox et al., 2018; Kiafar et al., 2017; Mehdizadeh et al., 2017). Nos momentos em que a ETP é superior à precipitação, há escassez de água para os cultivos. Dessa forma, com o aumento da ETP ocorre redução da água disponível para as atividades humanas e para os processos fisiológicos das plantas (Maček et al., 2018).

A ETP pode ser determinada por diversos métodos, podendo ser diretos e indiretos. Os métodos diretos apresentam maior precisão e são relativamente caros, enquanto os métodos indiretos se baseiam em fórmulas empíricas que combinam diferentes elementos meteorológicos (Trigo et al., 2018). As equações da ETP podem ter um desempenho diferente sob a mudança climática, assim levando a vários impactos sobre a produtividade das culturas agrícolas (Ramarohetra e Sultan, 2018).

A Organização das Nações Unidas para a Alimentação e Agricultura (FAO) sugeriu o método de PenmanMonteith (Allen et al., 1998), também conhecido como FAO56-PM. Esse método tem ótima precisão na estimativa de ETP (Mokhtari et al., 2018), bem eficiente em várias condições climáticas e em diversos locais (Maček et al., 2018; Medina et al., 2018; Peng et al., 2017). No entanto, esse método-padrão requer muitas variáveis climáticas (como temperatura do ar e umidade relativa, radiação solar e velocidade do vento) (Didari e Ahmadi, 2018).

Algumas variáveis de entrada nem sempre estão disponíveis ou não tem precisão e qualidade para um determinado local, principalmente em países em desenvolvimento (Mokhtari et al., 2018). Outro problema é a exigência de alto custo na instalação e manutenção de estações meteorológicas automatizadas (Valiantzas, 2018). No Brasil, a maioria das estações disponibilizam apenas os dados de temperatura do ar e precipitação pluvial, o que comprometem a aplicação do método padrão da FAO56PM (Santos et al., 2016).

No boletim da FAO-56 são dadas duas recomendações: o uso de métodos empíricos menos exigentes como, por exemplo, Hargreaves e Samani $(1982 ; 1985)$, esse método empírico requer apenas dados de temperatura média do ar e de radiação solar (Tomas-burguera et al., 2017; Valiantzas, 2018). A outra recomendação do boletim é que os métodos empíricos sejam ajustados para outras regiões por meio de medidas diretas de ETP (lisimétricas) e na indisponibilidade dessas medidas pode ser considerado o método da FAO56-PM como padrão (Allen et al., 1998; Santos et al., 2016).

Há métodos empíricos da ETP com entrada mínima de dados, a exemplo Thornthwaite (1948) e Camargo (1971) que são utilizados com mais frequência e requerem apenas a temperatura do ar (Santos et al., 2018; Gharbia et al., 2018; Ma et al., 2018; Moraes et al., 2018; Seong et al., 2018). Enquanto, Jensen-Haise (1963) e Makkink (1957) são baseados na radiação solar, os quais requerem apenas os dados de temperatura máxima e mínima do ar. A maioria dos métodos para estimar a ETP na literatura são em escala diária. Os principais métodos projetados em escala mensal são Camargo, Linacre, Hamon, Romanenko, Lungeon e Thornthwaite (Cunha et al., 2017b; Fernandes et al., 2010).

O conhecimento da precisão dos diversos modelos de ETP existentes em diferentes condições agronômicas e climáticas é bastante útil para o manejo da irrigação. A irrigação bem manejada garante aumento na produtividade e melhoria na qualidade dos produtos agrícolas. Encontrar um método que seja eficiente com o padrão da FAO56PM, e seja mais fácil de aplicar em campo é fundamental para a área de agrometeorologia. Os modelos indiretos são bastantes sensíveis de medir em sua escala apropriada e podem apresentar variabilidade espacial e temporal devido suas variáveis de entrada, além disso, esses modelos teve suas calibrações em regiões diferentes.

Portanto, objetivou-se comparar 18 métodos de estimativa de evapotranspiração potencial com o método padrão de Penman-Monteith em diferentes escalas de tempo para o Estado de Mato Grosso do Sul.

\section{Material e Métodos}

\section{1. Área de estudo e dados}

As séries históricas dos dados climáticos sequencias foram coletadas de 22 localidades do Estado de Mato Grosso do Sul, Brasil (Fig. 1 e Tabela 1). Os dados não passaram por nenhuma limpeza ou demais tratamentos preliminares, uma vez que esses apresentam consistência em suas informações.

Os dados climáticos foram provenientes da plataforma de dados NASA-POWER (2019) e obtidos na escala diária entre 1983 e 2018, com informações de radiação solar global $\left(\mathrm{MJ} \mathrm{m} \mathrm{m}^{-2} \mathrm{~d}^{-1}\right)$, temperatura média, máxima e mínima do ar $\left({ }^{\circ} \mathrm{C}\right)$, umidade relativa (\%), velocidade do vento $\left(\mathrm{m} \mathrm{s}^{-1}\right)$ e precipitação pluvial $(\mathrm{mm})$, esses 
Tabela 1 - Dados geográficos das localidades do Mato Grosso do Sul avaliadas.

\begin{tabular}{|c|c|c|c|}
\hline Cidades & Latitude & Longitude & Altitude $(\mathrm{m})$ \\
\hline Amambai & $23^{\circ} 06^{\prime} 15^{\prime \prime}$ & $55^{\circ} 13^{\prime} 33^{\prime \prime}$ & 480 \\
\hline Aral Moreira & $22^{\circ} 56^{\prime} 02^{\prime \prime}$ & $55^{\circ} 38^{\prime} 07^{\prime \prime}$ & 609 \\
\hline Bandeirantes & $19^{\circ} 55^{\prime} 04^{\prime \prime}$ & $54^{\circ} 21^{\prime} 50^{\prime \prime}$ & 629 \\
\hline Caarapó & $22^{\circ} 38^{\prime} 03^{\prime \prime}$ & $54^{\circ} 49^{\prime} 20^{\prime \prime}$ & 471 \\
\hline Camapuã & $19^{\circ} 31^{\prime} 53^{\prime \prime}$ & $54^{\circ} 02^{\prime} 38^{\prime \prime}$ & 409 \\
\hline Campo Grande & $20^{\circ} 26^{\prime} 34^{\prime \prime}$ & $54^{\circ} 38^{\prime} 47^{\prime \prime}$ & 532 \\
\hline Chapadão do Sul & $18^{\circ} 47^{\prime} 39^{\prime \prime}$ & $52^{\circ} 37^{\prime} 22^{\prime \prime}$ & 790 \\
\hline Costa Rica & $18^{\circ} 32^{\prime} 38^{\prime \prime}$ & $53^{\circ} 07^{\prime} 45^{\prime \prime}$ & 641 \\
\hline Coxim & $18^{\circ} 30^{\prime} 24^{\prime \prime}$ & $54^{\circ} 45^{\prime} 36^{\prime \prime}$ & 238 \\
\hline Douradina & $22^{\circ} 02^{\prime} 25^{\prime \prime}$ & $54^{\circ} 36^{\prime} 46^{\prime \prime}$ & 553 \\
\hline Dourados & $22^{\circ} 13^{\prime} 16^{\prime \prime}$ & $54^{\circ} 48^{\prime} 20^{\prime \prime}$ & 430 \\
\hline Itaporã & $22^{\circ} 04^{\prime} 44^{\prime \prime}$ & $54^{\circ} 47^{\prime} 22^{\prime \prime}$ & 390 \\
\hline Itaquiraí & $23^{\circ} 28^{\prime} 28^{\prime \prime}$ & $54^{\circ} 11^{\prime} 06^{\prime \prime}$ & 340 \\
\hline Laguna Carapã & $22^{\circ} 32^{\prime} 47^{\prime \prime}$ & $55^{\circ} 08^{\prime} 59^{\prime \prime}$ & 509 \\
\hline Maracaju & $21^{\circ} 36^{\prime} 52^{\prime \prime}$ & $55^{\circ} 10^{\prime} 06^{\prime \prime}$ & 384 \\
\hline Naviraí & $23^{\circ} 03^{\prime} 54^{\prime \prime}$ & $54^{\circ} 11^{\prime} 26^{\prime \prime}$ & 362 \\
\hline Nova Alvorada do Sul & $21^{\circ} 27^{\prime} 57^{\prime \prime}$ & $54^{\circ} 23^{\prime} 02^{\prime \prime}$ & 407 \\
\hline Ponta Porã & $22^{\circ} 32^{\prime} 10^{\prime \prime}$ & $55^{\circ} 43^{\prime} 32^{\prime \prime}$ & 655 \\
\hline Rio Brilhante & $21^{\circ} 48^{\prime} 07^{\prime \prime}$ & $54^{\circ} 32^{\prime} 47^{\prime \prime}$ & 312 \\
\hline São Gabriel do Oeste & $19^{\circ} 23^{\prime} 43^{\prime \prime}$ & $54^{\circ} 33^{\prime} 59^{\prime \prime}$ & 658 \\
\hline Sidrolândia & $20^{\circ} 55^{\prime} 55^{\prime \prime}$ & $54^{\circ} 57^{\prime} 41^{\prime \prime}$ & 484 \\
\hline Sonora & $17^{\circ} 34^{\prime} 37^{\prime \prime}$ & $54^{\circ} 45^{\prime} 28^{\prime \prime}$ & 442 \\
\hline
\end{tabular}

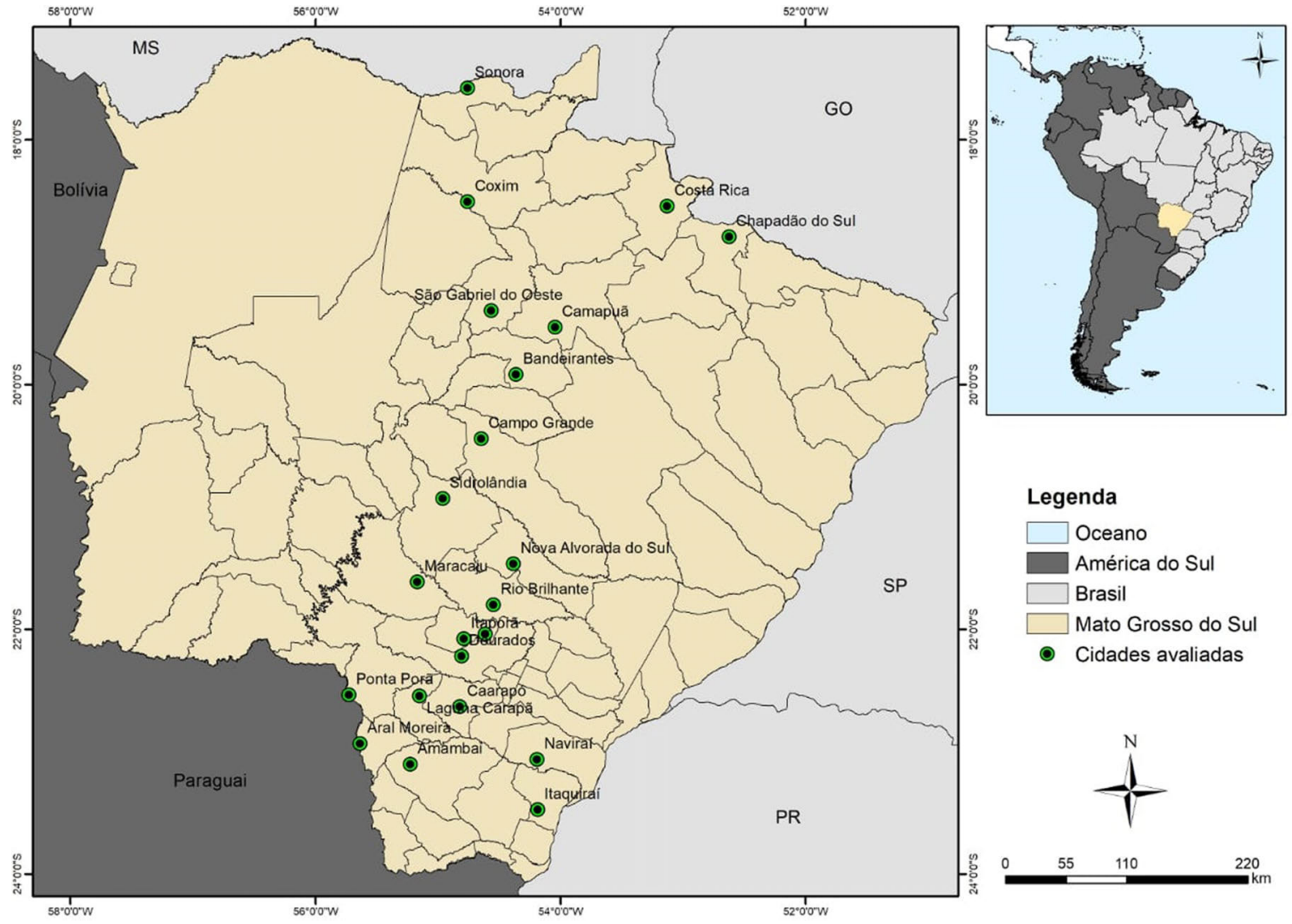

Figura 1 - Localização das estações meteorológicas do Mato Grosso do Sul, Brasil utilizadas no trabalho. 
dados são baseados em observações de satélites de grids globais de $0,5^{\circ}$ latitude-longitude. Com esses dados foram estimadas as evapotranspirações potenciais (ETPs) por 19 métodos em escalas diárias, semanais e mensais para a avaliação do desempenho desses métodos.

\subsection{Estimativa da ETP}

Os métodos testados para estimar a ETP para as localidades de Mato Grosso do Sul foram:

a. Penman e Monteith (Allen et al., 1998) (PM):

$$
\begin{gathered}
\text { EToPM }=\frac{0,408 \times s \times(R n-G)+\frac{\gamma \times 900 \times U_{2} \times(e s-e a)}{T+273}}{s+\gamma \times\left(1+0,34 \times U_{2}\right)} \\
s=\frac{4098 \times e s}{(T+273)^{2}} \quad e a=\frac{U R \times e s}{100} \quad e s=0,6108 \times e^{\frac{17,27 \times T}{237,3+T}}
\end{gathered}
$$

em que $s$ é a declividade da curva de pressão de vapor em relação à temperatura $\left(\mathrm{kPa}{ }^{\circ} \mathrm{C}\right) ; R n$ é o saldo de radiação diário $\left(\mathrm{MJ} \mathrm{m}^{-2} \mathrm{~d}^{-1}\right)$; $G$ é o fluxo total diário de calor no solo, assumindo-se valor igual a zero; $\gamma$ é o coeficiente psicrométrico (valor constante de $6,215.10^{-2} \mathrm{kPa}{ }^{\circ} \mathrm{C}$ ); $U_{2}$ é a velocidade do vento a $2 \mathrm{~m}$ de altura $\left(\mathrm{m} \mathrm{s}^{-1}\right)$; es é a pressão de saturação de vapor $(\mathrm{kPa})$; ea é a pressão atual de vapor $(\mathrm{kPa})$; e $T$ a temperatura média do ar $\left({ }^{\circ} \mathrm{C}\right)$.

b. Camargo (1971) (CAM):

$$
\begin{gathered}
E T o C=0,01 \times \frac{Q o}{2,45} \times T \times N D \\
h n=\operatorname{arcos}(-\tan \phi \times \tan \delta) \\
Q o=37,6 \times D R \times\left[\left(\frac{\pi}{180}\right) \times h n\right. \\
\times \operatorname{sen} \phi \times \operatorname{sen} \delta+\cos \phi \times \cos \delta \times \operatorname{sen} h n] \\
D R=1+0,33 \times \cos \left(360 \times \frac{N D A}{365}\right) \\
\delta=23,45 \times \operatorname{sen}\left[\left(\frac{360}{365}\right) \times N D A \times 80\right]
\end{gathered}
$$

em que $Q o$ é a irradiação solar extraterrestre $\left(\mathrm{MJ} \mathrm{m}^{-2} \mathrm{~d}^{-1}\right)$, $N D$ é o número de dias, $h n$ é a hora que ocorre o nascer do sol, $\phi$ é a latitude $\left(^{\circ}\right), \delta$ é a declinação solar $\left(^{\circ}\right), N D A$ é o dia Juliano, $D R$ é a distância relativa Terra-Sol.

c. Hargreaves (Hargreaves e Allen, 2003) (HAR):

$$
\begin{aligned}
E T o H & =0,0023 x(T n+17,8) \\
& x(\text { Tmax }-T \min )^{0,5} x Q o
\end{aligned}
$$

em que $T n$ é a temperatura média mensal $\left({ }^{\circ} \mathrm{C}\right)$, Tmax é a temperatura máxima diária $\left({ }^{\circ} \mathrm{C}\right)$ e $T$ min é a temperatura mínima diária $\left({ }^{\circ} \mathrm{C}\right)$. d. Priestley e Taylor (1972) (PT):

$$
\begin{gathered}
\text { EToPT }=1,26 \times W \times\left[\frac{(R n-G)}{2,45}\right] \\
\left\{\begin{array}{l}
W=0,407+0,0145 * T u, \text { para } 0{ }^{\circ} \mathrm{C}<\mathrm{T} \leq 16{ }^{\circ} \mathrm{C} \\
W=0,483+0,01 * T u, \text { para } 16{ }^{\circ} \mathrm{C}<\mathrm{T} \leq 32{ }^{\circ} \mathrm{C}
\end{array}\right.
\end{gathered}
$$

em que $W$ é o fator de peso dependente da temperatura e do coeficiente psicrométrico $\left({ }^{\circ} \mathrm{C}\right)$ e $T u$ é a temperatura do bulbo úmido $\left({ }^{\circ} \mathrm{C}\right)$.

e. Benevides e Lopez (1970) (BL):

$$
\begin{array}{r}
\text { EToBL }=1,21 \times 10^{\frac{7,5 \times T}{237,5+T}} \\
\times(1-0,01 \times U R)+0,21 \times T-2,3
\end{array}
$$

f. Jensen e Haise (1963) (JH):

$$
\text { EToJH }=\frac{Q g}{2,45} \times(0,078+0,052 \times T)
$$

em que Qg é a irradiação solar global $\left(\mathrm{MJ} \mathrm{m}^{-2} \mathrm{~d}^{-1}\right)$.

g. g) Tanner e Pelton (1960) (TP):

$$
\text { EToTP }=1,12 \times\left[\left(\frac{R n \times 100}{4,18}\right) / 59\right]-0,11
$$

h. Turc (1961) (TUR):

$$
\text { EToTUR }=0,013 \times\left(\frac{T_{\max }}{T_{\max }+15}\right) \times\left(\frac{Q_{g} \times 100}{4,18}+50\right)
$$

i. Hargreaves e Samani (1985) (HS):

$$
\begin{array}{r}
\text { EToHS }=0,0023 \times \frac{Q_{O}}{2,45} \\
\times\left(T_{\text {max }}-T_{\text {min }}\right)^{0,5} \times(T+17,8)
\end{array}
$$

j. Jobson (Bowie et al., 1985) (JOB):

$$
\text { ETоJOB }=3,01+1,13 \times U_{2} \times(e s-e a)
$$

k. Hamon (1961) (HAM):

$$
\begin{gathered}
\text { EToHAM }=0,55 \times\left(\frac{N}{12}\right)^{2} \times\left(\frac{4,95 \times e^{0,062 \times T}}{100}\right) \times 25,4 \\
N=\frac{2 \times h n}{15}
\end{gathered}
$$

em que $N$ é o fotoperíodo (horas). 
1. Makkink (1957) (MAK):

$$
\text { EToMAK }=0,61 \times W \times\left(\frac{Q_{g}}{2,45}\right)-0,12
$$

m. Linacre (1977) (LIN):

$$
\begin{gathered}
\text { EToLIN }=\frac{\frac{500 \times T_{m}}{100-\phi}+15 X(T-T o)}{80-T} \\
\text { To }=\frac{237,3 \times \log \left(\frac{e a}{0,0611}\right)}{7,5-\log \left(\frac{e a}{0,611}\right)} \\
T_{m}=T+0,006 \times h
\end{gathered}
$$

em que To é a temperatura de ponto de orvalho $\left({ }^{\circ} \mathrm{C}\right), T_{m}$ é a temperatura média ao nível do mar $\left({ }^{\circ} \mathrm{C}\right), h$ é a altitude (m).

n. Romanenko (1961) (ROM):

$$
\text { EToROM }=0,0018 \times(25+T)^{2} \times(100-U R)
$$

o. Kharrufa (1985) (KHA):

$$
\text { EToKHA }=0,34 \times p \times\left(T^{1,3}\right)
$$

em que $p$ é o índice fornecido por Doorenbos e Pruitt (1977).

p. Penman (1948) (PEN):

$$
\begin{gathered}
\text { EToPEN }=\frac{W \times R n+(1-W) \times \lambda E a}{2,45} \\
\lambda E a=6,43 \times\left(1+0,526 \times U_{2}\right) \times(e s-e a)
\end{gathered}
$$

em que $\lambda E$ Ea é a energia de evaporação de $\operatorname{ar}\left(\mathrm{MJ} \mathrm{m}^{-2} \mathrm{~d}^{-1}\right)$. q. Radiation (Doorenbos e Pruitt, 1977) (RAD):

$$
\begin{gathered}
\text { EToRAD }=c o+c l \times W \times\left(\frac{Q_{g}}{2,45}\right) \\
c l=a_{0}+a_{1}+U R+a_{2}+U_{2}+ \\
a_{3} \times U R \times U_{2}+a_{4} \times U R^{2} a_{5} \times U_{2}^{2}
\end{gathered}
$$

em que: co, cl são coeficientes de ajuste, co $=-0,3, \mathrm{a}_{0}=$ $1,0656, \mathrm{a}_{1}=-1,275 \times 10^{-3}, \mathrm{a}_{2}=4,4953 \times 10^{-2}, \mathrm{a}_{3}=2,033 \times$ $10^{-4}, a_{4}=-3,1508 \times 10^{-5}, a_{5}=-1,1026 \times 10^{-3}$.

r. Blaney e Criddle (1950) (BC):

$$
\begin{aligned}
E T o B C & =a+b \times p \times(0,46 \times T+8,13) \\
a & =0.043 \times U R_{\text {min }} \times\left(\frac{n}{N}\right) \times 1,41
\end{aligned}
$$

$$
\begin{aligned}
b= & a_{0}+a_{1} \times U R_{\text {min }}+a_{2} \times \frac{n}{N}+a_{3} \times U_{2} \\
& +a_{4} \times U R_{\text {min }} \times \frac{n}{N}+a_{5} \times U R_{\text {min }} \times U_{2}
\end{aligned}
$$

em que: $\mathrm{n}$ é a insolação (horas), URmín é a umidade relativa mínima diária (\%), $\mathrm{a}_{0}=0,81917, \mathrm{a}_{1}=-4,0922 \times 10^{-3}$, $a_{2}=1,0705, a_{3}=6,5649 \times 10^{-2}, a_{4}=-5,9684 \times 10^{-3}, a_{5}=$ $-5,967 \times 10^{-4}$.

s. Thornthwaite (1948) (THO):

$$
\begin{gathered}
\text { EToTHO }=\text { ETp } \times \text { Cor } \\
\text { Cor }=\left(\frac{N D}{30}\right) \times\left(\frac{N}{12}\right) \\
I=(0,2 \times T n)^{1,514}
\end{gathered}
$$

$$
\begin{aligned}
& \left\{\begin{array}{l}
E T p=-415,85+23,24 \times T-0,43 \times T^{2}, \\
\text { para } T \geq 26,5^{\circ} \mathrm{C} \\
E T p=16 \times\left(10 \times \frac{T}{I}\right)^{a}, \\
\text { para } 0{ }^{\circ} \mathrm{C} \leq T<26,5{ }^{\circ} \mathrm{C}
\end{array}\right. \\
& a=6,75 \times 10^{-7} \times I^{3} \times\left(-7,71 \times 10^{-5} \times I^{2}\right) \\
& +1,7912 \times 10^{-2} \times I+0,49239
\end{aligned}
$$

em que ETp é a evapotranspiração potencial média padrão $\left(\mathrm{mm} \mathrm{mês}^{-1}\right)$, I é o índice de calor mensal $\left({ }^{\circ} \mathrm{C}\right)$.

\subsection{Análises dos resultados}

A comparação entre os modelos de estimativa de ETP e o modelo FAO-56 PM foi realizada pelos índices estatísticos: acurácia e precisão. A acurácia consiste no quanto a estimativa está próxima do valor observado, e foi avaliada pelo MAPE (Erro absoluto percentual médio). A precisão é a capacidade do modelo repetir a estimativa foi avaliada pelo coeficiente de determinação ajustado $\left(\mathrm{R}^{2} \mathrm{aj}\right)$, segundo Cornell e Berger (1987).

$$
\begin{gathered}
\operatorname{MAPE}(\%)=\frac{\sum_{i=1}^{N}\left(\left|\frac{\text { ETPest }_{i}-\text { ETPobs }_{i}}{\text { ETPobs }_{i}}\right| \times 100\right)}{N} \\
R^{2} a j=\left[1-\frac{\left(1-R^{2}\right) \times(n-1)}{N-k-1}\right]
\end{gathered}
$$

em que ETPest ${ }_{i}$ é a Evapotranspiração Potencial estimada; ETPobs $_{\mathrm{i}}$ é a Evapotranspiração Potencial observada; N é o número de dados; e $\mathrm{k}$ o número de variáveis independentes na regressão.

Foi utilizada a Raiz do Erro Quadrático Médio que mede o grau de erro não simétrico entre os valores estima- 
dos e dados medidos/de referência (Moelestsi e Walker, 2012) e MBE (desvios das médias) indica o quanto o modelo foi subestimado (valor negativo) ou superestimado (valor positivo) (Eqs. (38) e (39), respectivamente).

$$
\begin{aligned}
R M S E & =\sqrt{\sum_{i=1}^{n} \frac{(\text { ETPest }- \text { ETPPM })^{2}}{N}} \\
M B E & =\frac{\sum_{i=1}^{N}(\text { ETPest }- \text { ETPPM })}{N-1}
\end{aligned}
$$

em que ETPest é a evapotranspiração potencial estimada, ETPPM é a evapotranspiração potencial por Penman e Monteith e N é a quantidades de dados.

\section{Resultados e Discussão}

\subsection{Variabilidade climática}

A precipitação da maior parte do Mato Grosso do Sul está entre 1500 e 1650 mm (Fig. 2.A). Nos municípios de Ponta Porã, Araí Moreira, Amambaí e Itaquiraí ocorreram as menores temperaturas do Estado do Mato Grosso do Sul. No oeste do estado do MS está localizado os maiores valores de ETP. Os maiores valores de armazenamento hídrico estão no centro ao sul do estado do MS. Enquanto, a deficiência hídrica, menores de $25 \mathrm{~mm}$, está localizada na maior parte do estado. Os maiores valores de excedente hídrico foram em Costa Rica, Chapadão do Sul, Itaquirai, Amambaí e Aral Moreira.

\subsection{ETP em escala diária}

Em todas as 22 localidades analisadas no Estado de Mato Grosso do Sul, o método de Jensen-Haise foi o que apresentou a menor acurácia em relação ao método Penman-Monteith para escala diária de ETP (Tabela 2). O fraco desempenho desse método baseado em radiação solar com MAPEs superiores a $68 \%$, está de acordo com os resultados encontrados no Irã (Tabari; Grismer; Trajkovic, 2013), Sérvia (Trajkovic e Kolakovic, 2009) e na Flórida (Irmak et al. 2003).

Para a localidade de Sonora foi verificado o maior erro absoluto percentual médio (MAPE) de $72,2 \%$ na

Tabela 2 - Desempenho estatístico dos métodos de ETP na escala diária em relação ao Método Penman-Monteith (PM), considerando a acurácia (Erro absoluto percentual médio, MAPE (\%)). (BC) Benevidez-Lopez, (BL) Blaney-Criddle, (CAM) Camargo, (HAM) Hamon, (HAR) Hargreaves, (HS) Hargreaves-Samani, (JH) Jensen-Haise, (JOB) Jobson, (KHA) Kharrufa, (LIN) Linacre, (MAK) Makkink, (PEN) Penman, (PT) Priestley-Taylor, (RAD)

\begin{tabular}{|c|c|c|c|c|c|c|c|c|c|c|c|c|c|c|c|c|c|c|}
\hline & $\mathrm{BL}$ & $\mathrm{BC}$ & AM & HAM & IAR & HS & $\mathrm{JH}$ & JOB & KHA & LIN & MAK & PEN & PT & RAD & ROM & $\mathrm{TP}$ & THO & TUR \\
\hline & 36,8 & 38,0 & 27,2 & 13,3 & 53,8 & 34,6 & 68,9 & 40,3 & 40,8 & 66,1 & 19,7 & 38,1 & & 51,1 & $34, .3$ & 42,3 & 23,8 & 29,0 \\
\hline Aral Moreira & 35,2 & 38,5 & 26,6 & 13,1 & 54,4 & 35,1 & 69,3 & 42,1 & 41,3 & 66,6 & 20,1 & 39,5 & 35,5 & 51,6 & 34,6 & 42,4 & 24,2 & 29,6 \\
\hline tes & 26,7 & 41,0 & 27,9 & 151 & 57,7 & 37,3 & 71,2 & 50,8 & 46,4 & & 22,8 & 45,3 & & 54,3 & 35,7 & 42,3 & 30,0 & 35,7 \\
\hline Caarapó & 30,9 & 39,7 & 26,3 & 14,0 & 55,9 & 36,4 & 70,2 & 48,8 & 43,5 & 67,6 & 23,1 & 43,6 & 37,7 & 54,4 & 32,6 & 43,7 & 25,8 & 32,9 \\
\hline Camapuã & 24,2 & 39,2 & 23,7 & 12,9 & 57,2 & 36,5 & 70,9 & 49,7 & 44,8 & 67,9 & 21,8 & 44,6 & 36,7 & 53,9 & 33,6 & 42,6 & 27,4 & 34,7 \\
\hline Campo Grande & 23,2 & 39,4 & 24,9 & 14,4 & 57,8 & 36,5 & 71,2 & 52,4 & 46,2 & 69,1 & 22,3 & 46,3 & 36,9 & 54,5 & 35,6 & 42,1 & 29,2 & 36,3 \\
\hline Chapadão do Sul & 25,1 & 40,1 & 22,9 & 12,0 & 57,0 & 37,6 & 70,9 & 47,4 & 43,9 & 68,0 & 20,9 & 43,0 & 37,1 & 53,1 & 33,6 & 43,9 & 26,1 & 32,7 \\
\hline Costa Rica & 22,5 & 40,6 & 23,0 & 12,0 & 57,9 & 38,1 & 71,4 & 49,4 & 45,2 & 68,5 & 21,3 & 44,2 & 37,3 & 53,6 & 33,0 & 43,8 & 27,7 & 34,1 \\
\hline & 18,7 & 38,4 & 23,3 & 15,5 & 58,9 & 36,0 & 71,6 & 51,1 & 47,8 & 69,6 & 18,9 & 44,5 & 34,7 & 52,4 & 37,6 & 39,5 & 30,6 & 37,2 \\
\hline Douradina & 29,7 & 38,0 & 26,2 & 13,9 & 55,4 & 34,8 & 69,7 & 47,8 & 43,0 & 67,4 & 22,2 & 43,3 & 36,7 & 53,9 & 33,4 & 42,7 & 25,5 & 33,1 \\
\hline Dourados & 29,7 & 38,1 & 26,3 & 14,0 & 55,4 & 34,9 & 69,8 & 47,9 & 43,1 & 67,3 & 22,4 & 43,3 & 36,7 & 54,0 & 33,5 & 42,7 & 25,7 & 33,2 \\
\hline & 29,6 & 38,1 & 26,2 & 13,9 & 55,4 & 35,0 & 69,8 & 47,9 & 43,1 & 67,2 & 22,4 & 43,3 & 36,7 & 54,0 & 33,5 & 42,7 & 25,6 & 33,2 \\
\hline Itaquirai & 31,9 & 39,4 & 27,4 & 14,5 & 55,5 & 35,9 & 69,9 & 46,9 & 43,3 & 67,3 & 22,1 & 42,0 & 36,8 & 53,3 & 32,9 & 43,1 & 25,6 & 32,3 \\
\hline Laguna & 33,2 & 39,9 & 26,2 & 13,3 & 55,6 & 36,5 & 70,0 & 47,1 & 42,8 & 67,2 & 22,7 & 42,6 & 37,6 & 53,9 & 33,0 & 43,8 & 25,5 & 31,9 \\
\hline Maracaju & 27,9 & 39,4 & 25,6 & 13,8 & 56,5 & 36,3 & 70,5 & 50,7 & 44,3 & 67,7 & 23,5 & 45,3 & 37,6 & 55,0 & 34,2 & 43,3 & 27,0 & 34,4 \\
\hline Naviraí & 31,9 & 39,3 & 27,1 & 14,4 & 55,5 & 35,8 & 69,9 & 46,8 & 43,2 & 67,2 & 22,0 & 41,9 & 36,8 & 53,3 & 32,7 & 43,0 & 25,3 & 32,3 \\
\hline $\begin{array}{l}\text { Nova Alvorada } \\
\text { Sul }\end{array}$ & 27,2 & 38,1 & 25,4 & 13,7 & 56,0 & 34,9 & 70,1 & 48,3 & 43,8 & 67,5 & 21,9 & 43,7 & 36,4 & 53,8 & 33,6 & 42,1 & 26,1 & 33,9 \\
\hline Ponta Porã & 31,7 & 40,7 & 26,4 & 13,4 & 56,3 & 37,3 & 70,5 & 49,5 & 43,7 & 68,0 & 23,5 & 44,4 & 38,1 & 54,7 & 33,9 & 44,2 & 26,6 & 32,7 \\
\hline Rio Brilhante & 27,9 & 37,6 & 25,7 & 14,1 & 55,7 & 34,5 & 69,8 & 48,4 & 43,5 & 67,2 & 22,2 & 43,8 & 36,4 & 54,0 & 34,6 & 42,1 & 25,8 & 34,0 \\
\hline Sidrolândia & 23,6 & 40,6 & 25,2 & 14,7 & 58,2 & 37,6 & 71,6 & 54,3 & 46,6 & 69,2 & 23,7 & 47,5 & 38,0 & 55,5 & 35,8 & 43,2 & 29,6 & 36,7 \\
\hline Sonora & 17,6 & 39,6 & 22,9 & 15,6 & 59,6 & 37,4 & 72,2 & 52,8 & 48,5 & 70,2 & 19,3 & 45,5 & 35,5 & 52,8 & 37,5 & 40,7 & 32,7 & 37,7 \\
\hline Max & 36,8 & 41,0 & 27,9 & 15,6 & 59,6 & 38,1 & 72,2 & 54,3 & 48,5 & 70,2 & 23,7 & 47,5 & 38,1 & 55,5 & 37,6 & 44,2 & 32,7 & 37,7 \\
\hline Min & 17,6 & 37.6 & 22.9 & 12.0 & 53,8 & 34.5 & 68,9 & 40,3 & 40,8 & 66,1 & 18,9 & 38,1 & 34,7 & 51,1 & 32,6 & 39,5 & 23.8 & 29,0 \\
\hline Média & 27,87 & 39,22 & 25,54 & 13,89 & 56,46 & 36,14 & 70,45 & 48,59 & 44,23 & 67,90 & 21,85 & 43,60 & 36,72 & 53,67 & 34,25 & 42,68 & 26,94 & 33,70 \\
\hline
\end{tabular}
Radiation, (ROM) Romanenko, (TP) Tanner-Pelton, (THO) Thornthwaite e (TUR) Turc.

Legenda: Baixo Alto 

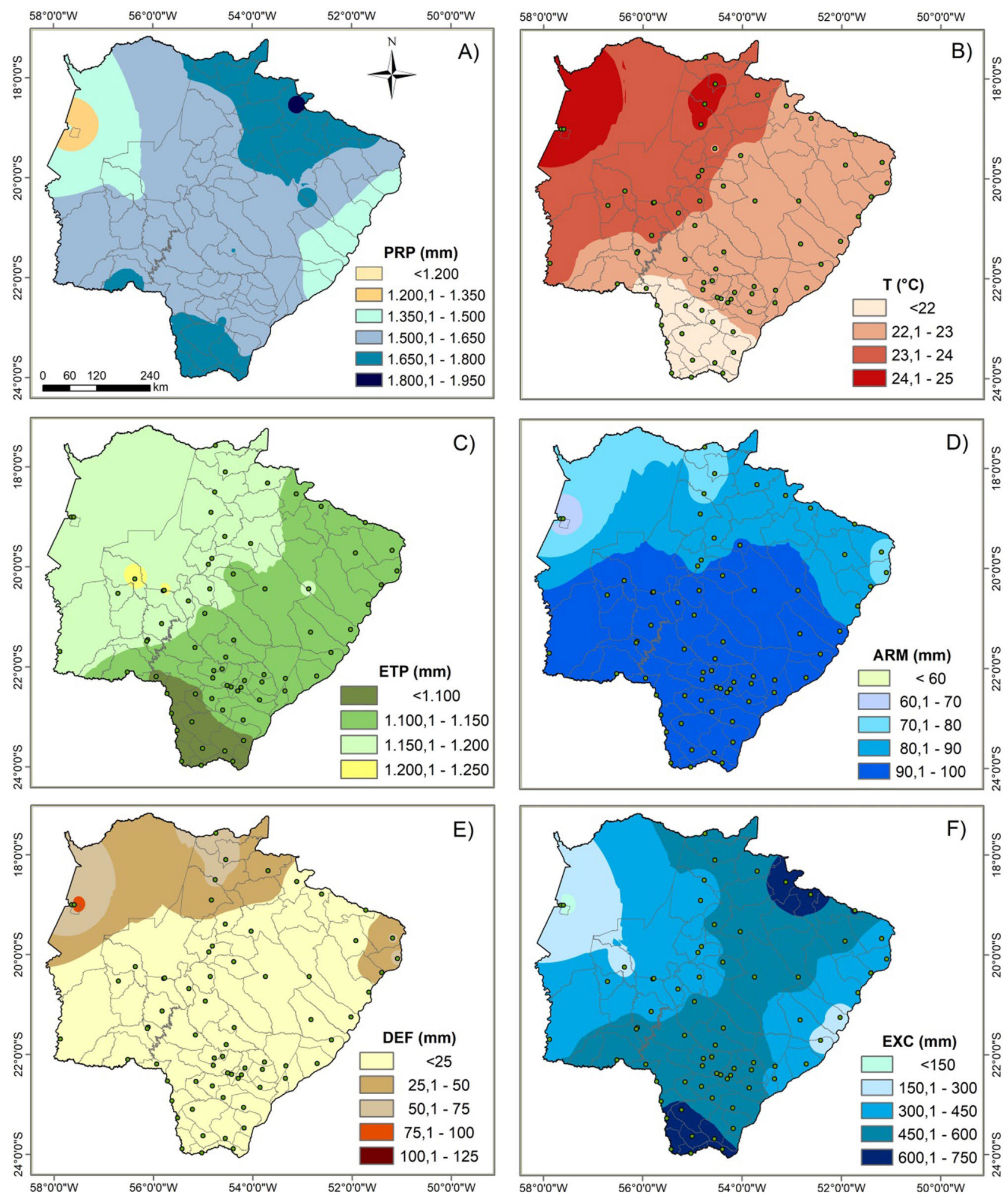

Figura 2 - Mapeamento da variabilidade climatica do estado do Mato Grosso do Sul.

escala diária de $\mathrm{JH}$ em relação a PM. O método de Hamon apresentou a maior acurácia em todas as regiões e nas cidades de Chapadão do Sul e Costa Rica se verificou o menor MAPE, de $12 \%$. Esse método é simples, confiável e requer apenas entradas de temperatura média, portanto, pode ser amplamente aplicada no tempo e no espaço (McCabe et al., 2015). O método de Hamon fornece estimativas próximas às de Thornthwaite, e tem sido utilizado 
em vários modelos hidrológicos (Almorox; Quej; Martí, 2015).

Outros métodos com baixo MAPE foram os de Makkink, Camargo e Thornthwaite, com valores de $21,85 \%, 25,54 \%$ e $26,94 \%$, respectivamente. Resultados similares para o método Makkink no Estado do Mato Grosso foram encontrados por Tanaka et al. (2016).

Os maiores coeficientes de determinação ajustado $\left(R^{2} a j\right)$ na escala diária foram para o método de PriestleyTaylor (PT) nas localidades de Amambai, Itaquirai e Naviraí, com 92\% (Tabela 3). O método Priestley-Taylor é baseado na radiação, o qual utiliza dados de entrada de temperatura do ar e radiação solar. O método de TannerPelton (TP), baseado na radiação, apresentou $\mathrm{R}^{2}$ aj igual a zero para todas as localidades. Esse método não demonstrou precisão na estimativa de ETP para escalas diárias.
Outros métodos pouco precisos foram os de CAM, ROM e JOB, cujos valores máximos de $\mathrm{R}^{2}$ aj foram de $0,11,0,16$ e 0,19 , respectivamente. Esses métodos foram pouco confiáveis para estimativa de ETP diária em Mato Grosso do Sul. Apesar da simplicidade de alguns métodos e da facilidade de adquirir os dados de entrada nas equações, esses métodos foram calibrados para ser utilizados em uma determinada região, mas devido sua alta dispersão de seus valores neste estudo não ocorreu uma calibração especifica para a região.

Os métodos com boa precisão foram os de MAK com $\mathrm{R}^{2}$ aj médio de 0,85 , HAR com 0,84 e RAD com 0,81 . $\mathrm{O} \mathrm{R}^{2}$ aj encontrado neste estudo para MAK foi superior ao encontrado por Caporusso e Rolim (2015).

Os métodos PT, HAR, HAM e MAK apresentaram alto desempenho para a escala diária (Fig. 3) no estado de

Tabela 3 - Desempenho estatístico dos métodos de ETP na escala diária em relação ao Método de Penman-Monteith considerando a precisão ( $\mathrm{R}^{2}$ aj). (BC) Benevidez-Lopez, (BL) Blaney-Criddle, (CAM) Camargo, (HAM) Hamon, (HAR) Hargreaves, (HS) Hargreaves-Samani, (JH) Jensen-Haise, (JOB) Jobson, (KHA) Kharrufa, (LIN) Linacre, (MAK) Makkink, (PEN) Penman, (PT) Priestley-Taylor, (RAD) Radiation, (ROM) Romanenko, (TP) Tanner-Pelton, (THO) Thornthwaite e (TUR) Turc.

\begin{tabular}{|c|c|c|c|c|c|c|c|c|c|c|c|c|c|c|c|c|c|c|}
\hline & BL & $\mathrm{BC}$ & CAM & HAM & HAR & HS & $\mathrm{JH}$ & JOB & KHA & LIN & MAK & PEN & PT & RAD & ROM & TP & THO & TUR \\
\hline Amabai & 0,27 & 0,27 & 0,11 & 0,74 & 0,84 & 0,62 & 0,64 & 0,01 & 0,27 & 0,26 & 0,85 & 0,77 & 0,92 & 0.81 & 0,04 & 0,00 & 0,38 & 0,70 \\
\hline Aral Moreira & 0,23 & 0,23 & 0,09 & 0,73 & 0,83 & 0,60 & 0,62 & 0,00 & 0,23 & 0,22 & 0,83 & 0,70 & 0,91 & 0.78 & 0,00 & 0,00 & 0,36 & 0,67 \\
\hline Bandeirantes & 0,02 & 0,02 & 0,02 & 0,65 & 0,81 & 0,55 & 0,54 & 0,19 & 0,02 & 0,02 & 0,73 & 0,32 & 0,90 & 0.60 & 0,16 & 0,00 & 0,10 & 0,62 \\
\hline Caarapo & 0,23 & 0,23 & 0,09 & 0,71 & 0,83 & 0,59 & 0,61 & 0,00 & 0,23 & 0,22 & 0,82 & 0,64 & 0,91 & 0.76 & 0,01 & 0,00 & 0,30 & 0,70 \\
\hline Camapua & 0,03 & 0,03 & 0,02 & 0,58 & 0,78 & 0,47 & 0,47 & 0,17 & 0,03 & 0,02 & 0,65 & 0,21 & 0,89 & 0.48 & 0,13 & 0,00 & 0,10 & 0,56 \\
\hline Campo Grande & 0,04 & 0,04 & 0,02 & 0,62 & 0,81 & 0,51 & 0,51 & 0,18 & 0,04 & 0,03 & 0,69 & 0,22 & 0,90 & 0.52 & 0,13 & 0,00 & 0,03 & 0,62 \\
\hline Chapadao do Sul & 0,02 & 0,02 & 0,02 & 0,55 & 0,74 & 0,45 & 0,44 & 0,12 & 0,02 & 0,01 & 0,64 & 0,21 & 0,89 & 0.47 & 0,09 & 0,00 & 0,20 & 0,50 \\
\hline Costa Rica & 002 & 0,02 & 0,02 & 0,57 & 0,77 & 0,46 & 0,44 & 0,12 & 0,02 & 0,01 & 0,63 & 0,17 & 0,89 & 0.44 & 0,09 & 0,00 & 0,16 & 0,51 \\
\hline Coxim & 0,00 & 0,00 & 0,00 & 0,49 & 0,76 & 0,42 & 0,41 & 0,16 & 0,00 & 0,00 & 0,57 & 0,12 & 0,90 & 0.37 & 0,13 & 0,00 & 0,08 & 0,55 \\
\hline Douradina & 0,18 & 0,18 & 0,07 & 0,69 & 0,82 & 0,58 & 0,59 & 0,01 & 0,18 & 0,17 & 0,80 & 0,59 & 0,91 & 0.72 & 0,00 & 0,00 & 0,13 & 0,69 \\
\hline Dourados & 0,18 & 0,18 & 0,07 & 0,69 & 0,82 & 0,58 & 0,59 & 0,01 & 0,18 & 0,17 & 0,80 & 0,59 & 0,91 & 0.72 & 0,00 & 0,00 & 0,13 & 0,69 \\
\hline Itaporã & 0,18 & 0,18 & 0,07 & 0,69 & 0,82 & 0,58 & 0,59 & 0,01 & 0,18 & 0,17 & 0,80 & 0,59 & 0,91 & 0.72 & 0,00 & 0,00 & 0,13 & 0,69 \\
\hline Itaquirai & 0,26 & 0,26 & 0,10 & 0,73 & 0,84 & 0,62 & 0,64 & 0,02 & 0,27 & 0,25 & 0,85 & 0,72 & 0,92 & 0.80 & 0,05 & 0,00 & 0,26 & 0,72 \\
\hline Laguna & 0,23 & 0,23 & 0,09 & 0,72 & 0,83 & 0,59 & 0,61 & 0,00 & 0,23 & 0,22 & 0,83 & 0,67 & 0,91 & 0.77 & 0,01 & 0,00 & 0,36 & 0,68 \\
\hline Maracaju & 0,14 & 0,14 & 0,06 & 0,67 & 0,81 & 0,56 & 0,57 & 0,06 & 0,14 & 0,12 & 0,77 & 0,45 & 0,90 & 0.66 & 0,02 & 0,00 & 0,14 & 0,66 \\
\hline Naviraí & 0,27 & 0,27 & 0,10 & 0,73 & 0,84 & 0,62 & 0,64 & 0,02 & 0,27 & 0,25 & 0,85 & 0,72 & 0,92 & 0.80 & 0,06 & 0,00 & 0,26 & 0,72 \\
\hline Nova Alvorada Sul & 0,11 & 0,11 & 0,04 & 0,65 & 0,81 & 0,55 & 0,55 & 0,07 & 0,11 & 0,10 & 0,77 & 0,48 & 0,91 & 0.67 & 0,04 & 0,00 & 0,09 & 0,66 \\
\hline Ponta Pora & 0,19 & 0,19 & 0,07 & 0,71 & 0,83 & 0,59 & 0,61 & 0,03 & 0,19 & 0,18 & 0,81 & 0,57 & 0,91 & 0.74 & 0,00 & 0,00 & 0,37 & 0,66 \\
\hline Rio Brilhante & 0,13 & 0,13 & 0,06 & 0,65 & 0,80 & 0,56 & 0,56 & 0,04 & 0,13 & 0,12 & 0,77 & 0,51 & 0,91 & 0.68 & 0,01 & 0,00 & 0,06 & 0,68 \\
\hline Sidrolandia & 0,07 & 0,07 & 0,03 & 0,64 & 0,81 & 0,53 & 0,53 & 0,15 & 0,07 & 0,06 & 0,71 & 0,26 & 0,90 & 0.55 & 0,09 & 0,00 & 0,07 & 0,63 \\
\hline Sonora & 0,00 & 0,00 & 0,00 & 0,45 & 0,74 & 0,39 & 0,37 & 0,13 & 0,00 & 0,00 & 0,53 & 0,08 & 0,90 & 0.33 & 0,10 & 0,00 & 0,05 & 0,51 \\
\hline Max & 0,27 & 0,27 & 0,11 & 0,74 & 0,84 & 0,62 & 0,64 & 0,19 & 0,27 & 0,26 & 0,85 & 0,77 & 0,92 & 0.81 & 0,16 & 0,00 & 0,38 & 0,72 \\
\hline Min & 0,00 & 0,00 & 0,00 & 0,45 & 0,74 & 0,39 & 0,37 & 0,00 & 0,00 & 0,00 & 0,53 & 0,08 & 0,89 & 0.33 & 0,00 & 0,00 & 0,03 & 0,50 \\
\hline Média & 0,13 & 0,13 & 0,05 & 0,65 & 0,81 & 0,54 & 0,55 & 0,07 & 0,13 & 0,12 & 0,75 & 0,46 & 0,91 & 0.64 & 0,06 & 0,00 & 0,18 & 0,64 \\
\hline
\end{tabular}



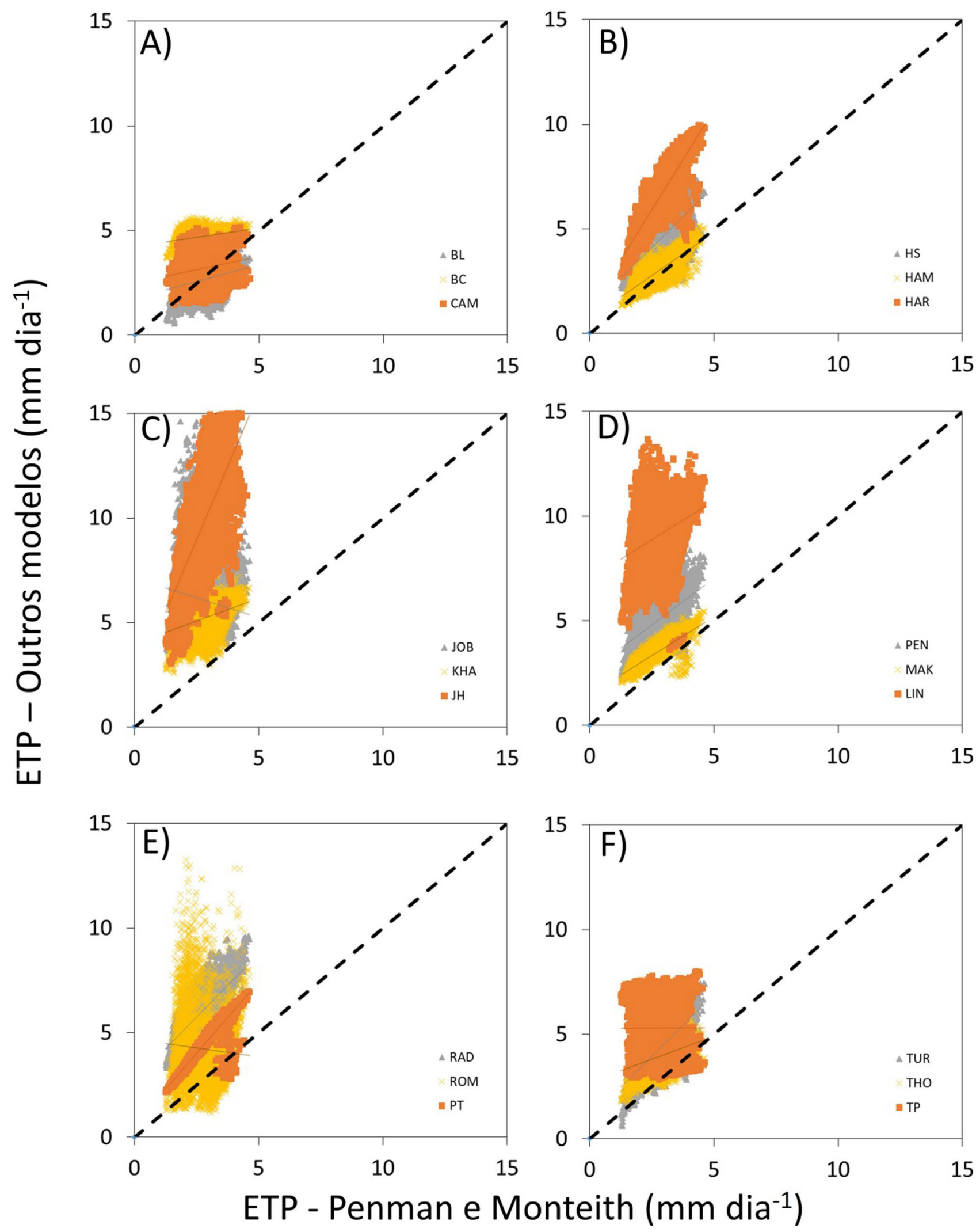

Figura 3 - Desempenho entre os dados Diários do modelo PM e os modelos (A)Benevidez-Lopez, Blaney-Criddle e Camargo, (B) Hamon, Hargreaves e, Hargreaves-Samani, (C) Jensen-Haise, (H) Jobson, (I) Kharrufa, (D) Linacre, Makkink e, Penman, (E) Priestley-Taylor, Radiation e, Romanenko, (F) Tanner-Pelton, Thornthwaite e Turc. 
Mato Grosso do Sul, apesar da simplicidade dessas equações. PT e MAK são baseados na radiação e os HAR e HAM são baseados na temperatura do ar. A calibração de alguns parâmetros dos métodos de estimativa da ETP pode melhorar seu desempenho consideravelmente (Carvalho et al., 2011).

Já os métodos CAM e THO não apresentaram boa precisão ( $R^{2}$ aj médio de 0,05 e 0,18 , respectivamente) na escala diária, apesar de apresentarem MAPE médio de $25,54 \%$ e $26,94 \%$, respectivamente. Além disso, o método de Camargo é derivado do método Thornthwaite.

O modelo com a maior variabilidade na escala diária foi TP (Fig. 3). Já o modelo que mais superestimou a evapotranspiração potencial foi JOB. Enquanto HAM e BL apresentaram os melhores desempenhos.

\subsection{ETP em escala semanal}

Os menores valores do erro (MAPE) nos métodos de ETP em escala semanal foram encontrados para HAM (Tabela 4). Em que $24,9 \%, 25,3 \%$ e $26,4 \%$ para as localidades de Costa Rica, Chapadão do Sul e Camapuã, respectivamente. Os métodos de MAK e CAM também apresentaram MAPE baixos, de 30,2\% e 31,3\%, respectivamente. Dentre esses três métodos, HAM e MAK apresentaram uma precisão média satisfatória de 0,65 e 0,75 , enquanto CAM obteve baixo $R^{2}$ aj de 0,05 .

Dentre os 18 métodos analisados para a escala semanal, vários métodos apresentaram baixa acurácia e precisão médias como, por exemplo, JH (MAPE de 69,8 e $\mathrm{R}^{2}$ aj de 0,55), LIN (MAPE de 68,3 e $\mathrm{R}^{2}$ aj de 0,12), TP (MAPE de 45,7 e R ${ }^{2}$ aj de 0,00), JOB (MAPE de 48,6 e $\mathrm{R}^{2}$ aj de 0,02) e ROM (MAPE de 45,0 e $\mathrm{R}^{2}$ aj de 0,06 )

Tabela 4 - Desempenho estatístico dos métodos de ETP na escala semanal em relação ao Método de Penman-Monteith, considerando a acurácia (Erro absoluto percentual médio, MAPE). (BC) Benevidez-Lopez, (BL) Blaney-Criddle, (CAM) Camargo, (HAM) Hamon, (HAR) Hargreaves, (HS) Hargreaves-Samani, (JH) Jensen-Haise, (JOB) Jobson, (KHA) Kharrufa, (LIN) Linacre, (MAK) Makkink, (PEN) Penman, (PT) Priestley-Taylor, (RAD) Radiation, (ROM) Romanenko, (TP) Tanner-Pelton, (THO) Thornthwaite e (TUR) Turc.

\begin{tabular}{|c|c|c|c|c|c|c|c|c|c|c|c|c|c|c|c|c|c|c|}
\hline & BL & $\mathrm{BC}$ & CAM & HAM & HAR & HS & JH & JOB & KHA & LIN & MAK & PEN & PT & RAD & ROM & TP & THO & TUR \\
\hline Amabai & 55,7 & 46,1 & 38,1 & 29,0 & 58,5 & 42,8 & 68,9 & 40,3 & 48,2 & 67,2 & 32,6 & 46,7 & 44,6 & 56,7 & 50,2 & 45,4 & 35,6 & 42,3 \\
\hline Aral Moreira & 53,4 & 46,4 & 37,2 & 28,4 & 58,7 & 43,0 & 69,1 & 42,1 & 48,4 & 67,5 & 32,7 & 47,4 & 44,7 & 56,9 & 48,4 & 45,4 & 35,6 & 40,1 \\
\hline Bandeirantes & 42,2 & 48,3 & 37,3 & 28,9 & 61,2 & 44,7 & 70,6 & 50,8 & 52,2 & 69,4 & 34,9 & 51,8 & 45,8 & 58,9 & 44,8 & 45,3 & 39,6 & 44,8 \\
\hline Caarapo & 48,3 & 47,3 & 36,4 & 28,6 & 59,9 & 44,0 & 69,8 & 48,8 & 50,1 & 68,3 & 34,9 & 50,5 & 46,3 & 58,9 & 45,6 & 46,4 & 36,7 & 42,7 \\
\hline Camapua & 39,4 & 46,2 & 33,1 & 26,4 & 60,1 & 43,2 & 69,9 & 49,7 & 50,3 & 68,1 & 33,1 & 50,4 & 44,8 & 57,9 & 42,4 & 45,6 & 36,7 & 43,0 \\
\hline Campo Grande & 38,2 & 46,4 & 34,1 & 27,7 & 60,8 & 43,3 & 70,3 & 52,4 & 51,6 & 69,1 & 33,9 & 52,3 & 45,1 & 58,7 & 45,4 & 45,1 & 38,3 & 44,7 \\
\hline Chapadao do Sul & 40,1 & 46,8 & 32,0 & 25,3 & 59,8 & 43,9 & 69,8 & 47,4 & 49,3 & 68,0 & 31,8 & 48,4 & 44,9 & 56,8 & 41,4 & 46,8 & 35,1 & 40,7 \\
\hline Costa Rica & 36,9 & 47,2 & 31,8 & 24,9 & 60,4 & 44,3 & 70,2 & 49,4 & 50,2 & 68,3 & 32,1 & 49,2 & 45,1 & 57,1 & 39,6 & 46,8 & 36,3 & 41,9 \\
\hline Coxim & 31,7 & 45,5 & 32,0 & 27,2 & 61,2 & 42,6 & 70,4 & 51,1 & 52,2 & 69,1 & 30,2 & 49,4 & 43,0 & 56,2 & 41,7 & 43,3 & 38,3 & 44,5 \\
\hline Douradina & 46,8 & 45,8 & 36,4 & 28,5 & 59,3 & 42,7 & 69,3 & 47,8 & 49,5 & 68,1 & 34,2 & 50,1 & 45,5 & 58,4 & 45,2 & 45,6 & 35,5 & 42,6 \\
\hline Dourados & 46,7 & 46,0 & 36,6 & 28,6 & 59,4 & 42,8 & 69,4 & 47,9 & 49,7 & 68,0 & 34,3 & 50,2 & 45,6 & 58,5 & 45,3 & 45,6 & 35,7 & 42,8 \\
\hline Itaporã & 46,6 & 46,0 & 36,4 & 28,5 & 59,4 & 42,8 & 69,4 & 47,9 & 49,7 & 67,9 & 34,3 & 50,1 & 45,5 & 58,5 & 45,2 & 45,6 & 35,6 & 42,8 \\
\hline Itaquirai & 49,8 & 47,2 & 37,7 & 29,5 & 59,7 & 43,8 & 69,7 & 46,9 & 50,1 & 68,2 & 34,5 & 49,7 & 45,8 & 58,4 & 47,1 & 45,9 & 36,5 & 42,6 \\
\hline Laguna & 50,9 & 47,4 & 36,3 & 28,2 & 59,5 & 44,1 & 69,6 & 47,1 & 49,4 & 67,9 & 34,6 & 49,7 & 46,2 & 58,5 & 46,5 & 46,5 & 36,4 & 41,7 \\
\hline Maracaju & 44,2 & 46,8 & 35,4 & 27,9 & 60,1 & 43,6 & 69,9 & 50,7 & 50,3 & 68,2 & 35,1 & 51,6 & 46,1 & 59,2 & 45,2 & 46,2 & 36,8 & 43,5 \\
\hline Naviraí & 49,7 & 47,0 & 37,4 & 29,3 & 59,7 & 43,6 & 69,6 & 46,8 & 50,0 & 68,1 & 34,3 & 49,6 & 45,8 & 58,3 & 47,0 & 45,9 & 36,2 & 42,5 \\
\hline Nova Alvorada Sul & 43,7 & 45,6 & 35,3 & 28,0 & 59,6 & 42,4 & 69,5 & 48,3 & 50,0 & 68,0 & 33,8 & 50,6 & 45,0 & 58,3 & 45,5 & 45,1 & 36,2 & 43,2 \\
\hline Ponta Pora & 48,7 & 48,0 & 36,3 & 27,9 & 60,1 & 44,7 & 70,0 & 49,5 & 50,1 & 68,5 & 35,3 & 51,0 & 46,6 & 59,1 & 45,7 & 46,8 & 37,2 & 42,2 \\
\hline Rio Brilhante & 44,4 & 45,4 & 35,9 & 28,4 & 59,4 & 42,2 & 69,4 & 48,4 & 49,8 & 67,8 & 34,1 & 50,5 & 45,1 & 58,5 & 45,9 & 45,2 & 35,6 & 43,3 \\
\hline Sidrolandia & 38,8 & 47,3 & 34,2 & 27,9 & 61,2 & 44,2 & 70,6 & 54,3 & 51,9 & 69,2 & 35,1 & 53,4 & 46,1 & 59,6 & 46,0 & 46,0 & 38,7 & 45,1 \\
\hline Sonora & 30,4 & 46,3 & 31,3 & 27,0 & 61,6 & 43,5 & 70,7 & 52,8 & 52,6 & 69,5 & 30,3 & 49,9 & 43,4 & 56,3 & 41,4 & 44,3 & 40,2 & 44,6 \\
\hline Max & 55,7 & 48,3 & 38,1 & 29,5 & 61,6 & 44,7 & 70,7 & 54,3 & 52,6 & $69, .5$ & 35,3 & 53,4 & 46,6 & 59,6 & 50,2 & 46,8 & 40,2 & 45,1 \\
\hline Min & 30,4 & 45,4 & 31,3 & 24,9 & 58,5 & 42,2 & 68,9 & 40,3 & 48,2 & 67,2 & 30,2 & 46,7 & 43,0 & 56,2 & 39,6 & 43,3 & 35,1 & 40,1 \\
\hline Média & 44,1 & 46,6 & 35,3 & 27,9 & 60,0 & 43,4 & 69,8 & 48,6 & 50,3 & 68,3 & 33,6 & 50,1 & 45,3 & 58,1 & 45,0 & 45,7 & 36,8 & 42,9 \\
\hline
\end{tabular}

Legenda: Baixo Alto 
(Tabelas 4 e 5), por isso esses métodos não são recomendados para o Estado de Mato Grosso do Sul.

Os diferentes resultados encontrados nesses trabalhos provavelmente estão ligados aos fatores climáticos e de altitude. Os métodos dependem da disponibilidade de dados locais, precisão exigida e/ou escala temporal, e dessa forma, para adequá-los a uma condição específica, muitos deles passam por calibrações (Carvalho et al., 2011).

Alguns métodos aliaram boa acurácia e precisão para a escala semanal (Fig. 4). HAM e MAK apresentaram baixos erros médios de 27,9 e 33,6, e boas precisões com $\mathrm{R}^{2}$ aj de 0,65 e 0,75 , respectivamente. Já PT, apesar da alta precisão $\left(\mathrm{R}^{2}\right.$ aj de 0,91$)$, obteve um MAPE relativamente alto $(45,3 \%)$. Isso aconteceu também para o método HAR que apresentou $\mathrm{R}^{2}$ aj médio de 0,81 e MAPE médio de $60 \%$.

O modelo CAM apresentou melhor desempenho quando comparado com os demais (Fig. 4). A evapotranspiração potencial semanal pelo método de $\mathrm{JH}$ foi o que mais superestimou nesta escala, enquanto que o método de BL foi o que subestimou a ETP.

\subsection{ETP em escala mensal}

HAM obteve maior acurácia em relação ao PenmanMonteith (PM) para ETP na escala mensal em todas as 22 localidades analisadas em Mato Grosso do Sul (Tabela 6). Para HAM o menor MAPE foi de 11,95\% em Chapadão do Sul e o maior de $15,64 \%$ em Sonora, a média ficou em $13,88 \%$. Outro método com boa acurácia foi MAK, com

Tabela 5 - Desempenho estatístico dos métodos de ETP na escala semanal em relação ao Penman-Monteith, considerando a precisão (R2 aj). (BC) Benevidez-Lopez, (BL) Blaney-Criddle, (CAM) Camargo, (HAM) Hamon, (HAR) Hargreaves, ( HS) Hargreaves-Samani, (JH) Jensen-Haise, (JOB) Jobson, (KHA) Kharrufa, (LIN) Linacre, (MAK) Makkink, (PEN) Penman, (PT) Priestley-Taylor, (RAD) Radiation, (ROM) Romanenko, (TP) Tanner-Pelton, (THO) Thornthwaite e (TUR) Turc.

\begin{tabular}{|c|c|c|c|c|c|c|c|c|c|c|c|c|c|c|c|c|c|c|}
\hline & BL & $\mathrm{BC}$ & CAM & HAM & HAR & HS & JH & JOB & KHA & LIN & MAK & PEN & PT & RAD & ROM & $\mathrm{TP}$ & $\mathrm{THO}$ & TUR \\
\hline Amabai & 0,27 & 0,27 & 0,11 & 0,74 & 0,84 & 0,62 & 0,64 & 0,05 & 0,27 & 0,26 & 0,85 & 0,77 & 0,92 & 0,81 & 0,04 & 0,00 & 0,38 & 0,70 \\
\hline Aral Moreira & 0,23 & 0,23 & 0,09 & 0,73 & 0,83 & 0,60 & 0,62 & 0,03 & 0,23 & 0,22 & 0,83 & 0,70 & 0,91 & 0,78 & 0,00 & 0,00 & 0,36 & 0,67 \\
\hline Bandeirantes & 0,02 & 0,02 & 0,02 & 0,65 & 0,81 & 0,55 & 0,54 & 0,00 & 0,02 & 0,02 & 0,73 & 0,32 & 0,90 & 0,60 & 0,16 & 0,00 & 0,10 & 0,62 \\
\hline Caarapo & 0,23 & 0,23 & 0,09 & 0,71 & 0,83 & 0,59 & 0,61 & 0,04 & 0,23 & 0,22 & 0,82 & 0,64 & 0,91 & 0,76 & 0,01 & 0,00 & 0,30 & 0,70 \\
\hline Camapua & 0,03 & 0,03 & 0,02 & 0,58 & 0,78 & 0,47 & 0,47 & 0,00 & 0,03 & 0,02 & 0,65 & 0,21 & 0,89 & 0,48 & 0,13 & 0,00 & 0,10 & 0,56 \\
\hline Campo Grande & 0,04 & 0,04 & 0,02 & 0,62 & 0,81 & 0,51 & 0,51 & 0,00 & 0,04 & 0,03 & 0,69 & 0,22 & 0,90 & 0,52 & 0,13 & 0,00 & 0,03 & 0,62 \\
\hline Chapadao do Sul & 0,02 & 0,02 & 0,02 & 0,55 & 0,74 & 0,45 & 0,44 & 0,01 & 0,02 & 0,01 & 0,64 & 0,21 & 0,89 & 0,47 & 0,09 & 0,00 & 0,20 & 0,50 \\
\hline Costa Rica & 0,02 & 0,02 & 002 & 0,57 & 0,77 & 0,46 & 0,44 & 0,01 & 0,02 & 0,01 & 0,63 & 0,17 & 0,89 & 0,44 & 0,09 & 0,00 & 0,16 & 0,51 \\
\hline Coxim & 0,00 & 0,00 & 0,00 & 0,49 & 0,76 & 0,42 & 0,41 & 0,01 & 0,00 & 0,00 & 0,57 & 0,12 & 0,90 & 0,37 & 0,13 & 0,00 & 0,08 & 0,55 \\
\hline Douradina & 0,18 & 0,18 & 0,07 & 0,69 & 0,82 & 0,58 & 0,59 & 0,02 & 0,18 & 0,17 & 0,80 & 0,59 & 0,91 & 0,72 & 0,00 & 0,00 & 0,13 & 0,69 \\
\hline Dourados & 0,18 & 0,18 & 0,07 & 0,69 & 0,82 & 0,58 & 0,59 & 0,02 & 0,18 & 0,17 & 0,80 & 0,59 & 0,91 & 0,72 & 0,00 & 0,00 & 0,13 & 0,69 \\
\hline Itaporã & 0,18 & 0,18 & 0,07 & 0,69 & 0,82 & 0,58 & 0,59 & 0,02 & 0,18 & 0,17 & 0,80 & 0,59 & 0,91 & 0,72 & 0,00 & 0,00 & 0,13 & 0,69 \\
\hline Itaquirai & 0,26 & 0,26 & 0,10 & 0,73 & 0,84 & 0,62 & 0,64 & 0,05 & 0,27 & 0,25 & 0,85 & 0,72 & 0,92 & 0,80 & 0,05 & 0,00 & 0,26 & 0,72 \\
\hline Laguna & 0,23 & 0,23 & 0,09 & 0,72 & 0,83 & 0,59 & 0,61 & 0,03 & 0,23 & 0,22 & 0,83 & 0,67 & 0,91 & 0,77 & 0,01 & 0,00 & 0,36 & 0,68 \\
\hline Maracaju & 0,14 & 0,14 & 0,06 & 0,67 & 0,81 & 0,56 & 0,57 & 0,01 & 0,14 & 0,12 & 0,77 & 0,45 & 0,90 & 0,66 & 0,02 & 0,00 & 0,14 & 0,66 \\
\hline Naviraí & 0,27 & 0,27 & 0,10 & 0,73 & 0,84 & 0,62 & 0,64 & 0,05 & 0,27 & 0,25 & 0,85 & 0,72 & 0,92 & 0,80 & 0,06 & 0,00 & 0,26 & 0,72 \\
\hline Nova Alvorada Sul & 0,11 & 0,11 & 0,04 & 0,65 & 0,81 & 0,55 & 0,55 & 0,01 & 0,11 & 0,10 & 0,77 & 0,48 & 0,91 & 0,67 & 0,04 & 0,00 & 0,09 & 0,66 \\
\hline Ponta Pora & 0,19 & 0,19 & 0,07 & 0,71 & 0,83 & 0,59 & 0,61 & 0,02 & 0,19 & 0,18 & 0,81 & 0,57 & 0,91 & 0,74 & 0,00 & 0,00 & 0,37 & 0,66 \\
\hline Rio Brilhante & 0,13 & 0,13 & 0,06 & 0,65 & 0,80 & 0,56 & 0,56 & 0,01 & 0,13 & 0,12 & 0,77 & 0,51 & 0,91 & 0,68 & 0,01 & 0,00 & 0,06 & 0,68 \\
\hline Sidrolandia & 0,07 & 0,07 & 0,03 & 0,64 & 0,81 & 0,53 & 0,53 & 0,00 & 0,07 & 0,06 & 0,71 & 0,26 & 0,90 & 0,55 & 0,09 & 0,00 & 0,07 & 0,63 \\
\hline Sonora & 0,00 & 0,00 & 0,00 & 0,45 & 0,74 & 0,39 & 0,37 & 0,01 & 0,00 & 0,00 & 0,53 & 0,08 & 0,90 & 0,33 & 0,10 & 0,00 & 0,05 & 0,51 \\
\hline Max & 0,27 & 0,27 & 0,11 & 0,74 & 0,84 & 0,62 & 0,64 & 0,05 & 0,27 & 0,26 & 0,85 & 0,77 & 0,92 & 0,81 & 0,16 & 0,00 & 0,38 & 0,72 \\
\hline Min & 0,00 & 0,00 & 0,00 & 0,45 & 0,74 & 0,39 & 0,37 & 0,00 & 0,00 & 0,00 & 0,53 & 0,08 & 0,89 & 0,33 & 0,00 & 0,00 & 0,03 & 0,50 \\
\hline Média & 0,13 & 0,13 & 0,05 & 0,65 & 0,81 & 0,54 & 0,55 & 0,02 & 0,13 & 0,12 & 0,75 & 0,46 & 0,91 & 0,64 & 0,06 & 0,00 & 0,18 & 0,64 \\
\hline
\end{tabular}

Legenda: Baixo Alto 

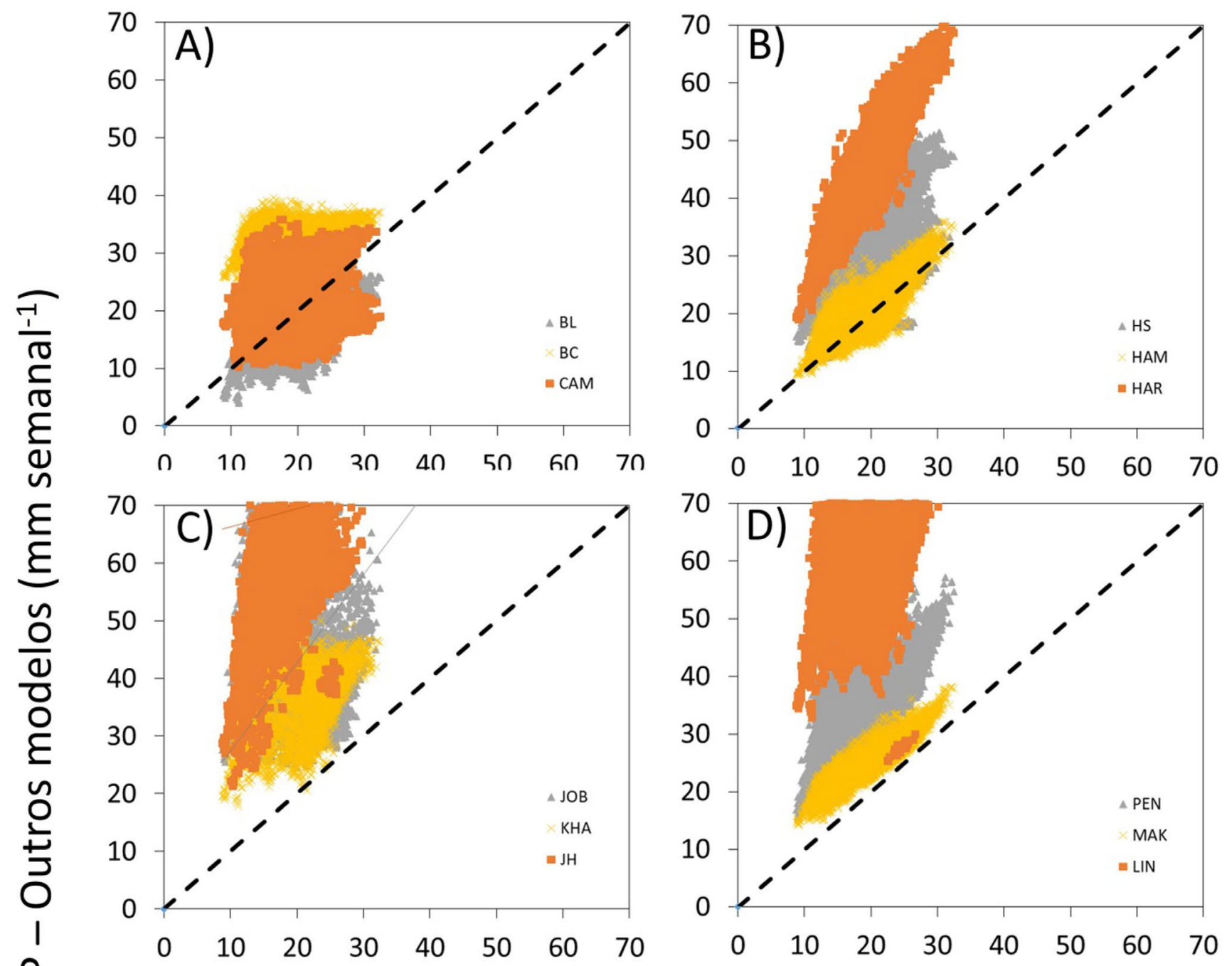

는
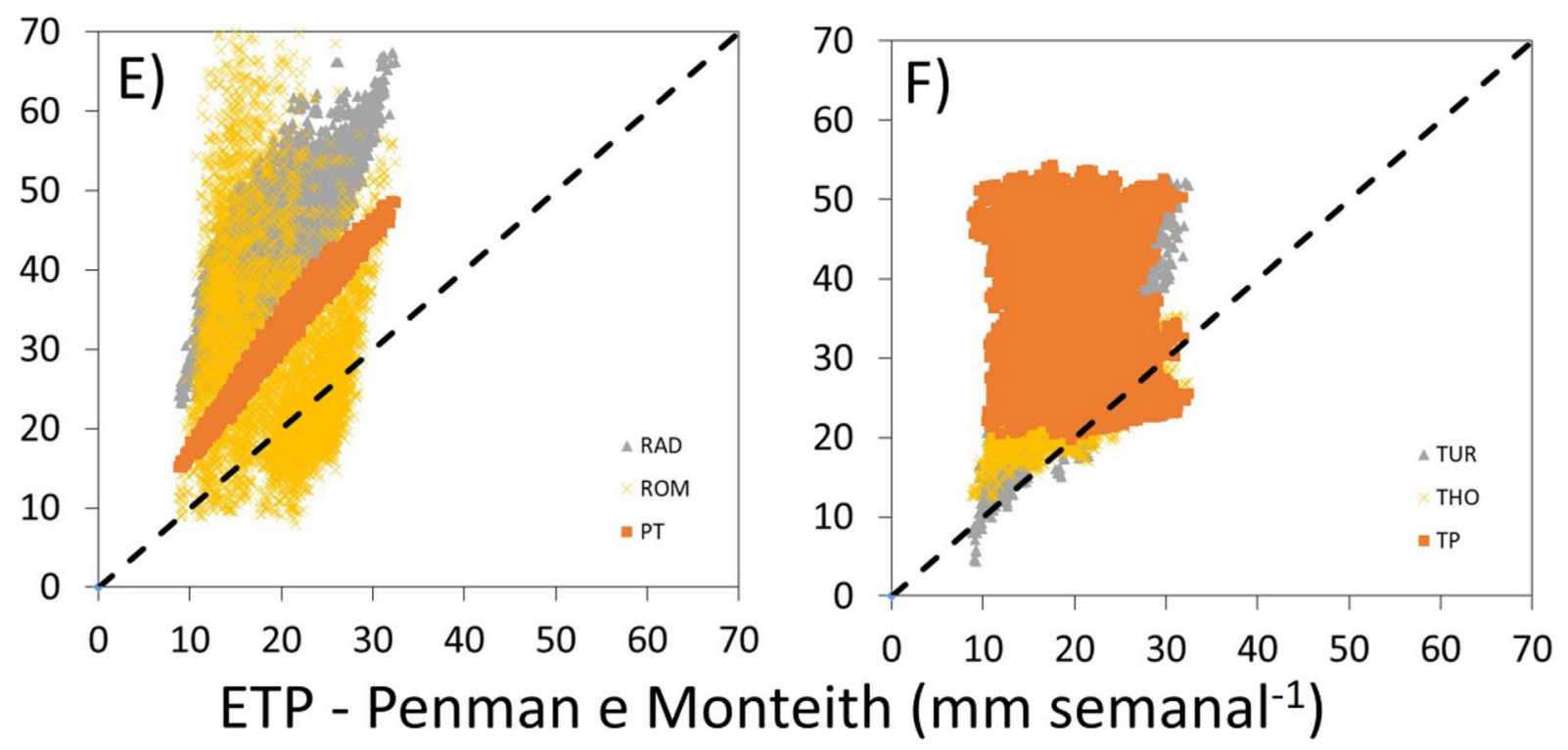

Figura 4 - Desempenho entre os dados semanais do modelo PM e os modelos (A) Benevidez-Lopez, Blaney-Criddle e Camargo, (B) Hamon, Hargreaves e, Hargreaves-Samani, (C) Jensen-Haise, (H) Jobson, (I) Kharrufa, (D) Linacre, Makkink e, Penman, (E) Priestley-Taylor, Radiation e, Romanenko, (F) Tanner-Pelton, Thornthwaite e Turc. 
Tabela 6 - Desempenho estatístico dos métodos de ETP na escala mensal em relação ao Método Penman-Monteith, considerando a acurácia (Erro absoluto percentual médio, MAPE). (BC) Benevidez-Lopez, (BL) Blaney-Criddle, (CAM) Camargo, (HAM) Hamon, (HAR) Hargreaves, (HS) HargreavesSamani, (JH) Jensen-Haise, (JOB) Jobson, (KHA) Kharrufa, (LIN) Linacre, (MAK) Makkink, (PEN) Penman, (PT) Priestley-Taylor, (RAD) Radiation, (ROM) Romanenko, (TP) Tanner-Pelton, (THO) Thornthwaite e (TUR) Turc.

\begin{tabular}{|c|c|c|c|c|c|c|c|c|c|c|c|c|c|c|c|c|c|c|}
\hline & BL & $\mathrm{BC}$ & CAM & HAM & HAR & HS & $\mathrm{JH}$ & $\mathrm{JOB}$ & KHA & LIN & MAK & PEN & PT & RAD & ROM & ТP & THO & TUR \\
\hline Amabai & 36,83 & 38,04 & 27,20 & 13,35 & 53,83 & 34,58 & 68,89 & 40,34 & 40,79 & 66,07 & 19,69 & 38,06 & 35,22 & 51,09 & 34,31 & 42,28 & 23,85 & 40,45 \\
\hline Aral Moreira & 35,25 & 38,46 & 26,58 & 13,08 & 54,40 & 35,09 & 69,26 & 42,12 & 41,32 & 66,62 & 20,08 & 39,47 & 35,49 & 51,60 & 34,58 & 42,39 & 24,17 & 29,62 \\
\hline Bandeirantes & 26,66 & 40,97 & 27,86 & 15,06 & 57,65 & 37,26 & 71,21 & 50,82 & 46,39 & 69,11 & 22,84 & 45,34 & 36,80 & 54,29 & 35,66 & 42,26 & 30,03 & 35,74 \\
\hline Caarapo & 30,91 & 39,74 & 26,34 & 13,96 & 55,94 & 36,38 & 70,20 & 48,81 & 43,55 & 67,60 & 23,05 & 43,62 & 37,68 & 54,36 & 32,64 & 43,67 & 25,79 & 32,94 \\
\hline Camapua & 24,22 & 39,20 & 23,70 & 12,94 & 57,18 & 36,52 & 70,89 & 49,66 & 44,77 & 67,92 & 21,80 & 44,64 & 36,75 & 53,93 & 33,55 & 42,63 & 27,44 & 34,66 \\
\hline Campo Grande & 23,24 & 39,40 & 24,87 & 14,38 & 57,85 & 36,50 & 71,23 & 52,43 & 46,21 & 69,11 & 22,31 & 46,26 & 36,89 & 54,48 & 35,61 & 42,08 & 29,19 & 36,32 \\
\hline Chapadao do Sul & 25,07 & 40,10 & 22,88 & 11,95 & 57,04 & 37,63 & 70,94 & 47,40 & 43,93 & 68,05 & 20,86 & 43,04 & 37,07 & 53,08 & 33,58 & 43,89 & 26,10 & 32,66 \\
\hline Costa Rica & 22,53 & 40,55 & 22,97 & 12,00 & 57,87 & 38,12 & 71,42 & 49,38 & 45,23 & 68,50 & 21,27 & 44,19 & 37,31 & 53,56 & 33,02 & 43,79 & 27,68 & 34,13 \\
\hline Coxim & 18,65 & 38,44 & 23,34 & 15,46 & 58,87 & 36,04 & 71,64 & 51,10 & 47,81 & 69,57 & 18,92 & 44,53 & 34,68 & 52,36 & 37,62 & 39,52 & 30,59 & 37,23 \\
\hline Douradina & 29,73 & 37,99 & 26,17 & 13,88 & 55,35 & 34,83 & 69,71 & 47,78 & 43,00 & 67,43 & 22,22 & 43,29 & 36,74 & 53,88 & 33,41 & 42,66 & 25,52 & 33,08 \\
\hline Dourados & 29,70 & 38,14 & 26,32 & 13,96 & 55,44 & 34,95 & 69,77 & 47,89 & 43,14 & 67,28 & 22,37 & 43,34 & 36,75 & 53,96 & 33,50 & 42,66 & 25,70 & 33,21 \\
\hline Itaporã & 29,65 & 38,12 & 26,21 & 13,93 & 55,45 & 34,96 & 69,78 & 47,89 & 43,12 & 67,18 & 22,38 & 43,33 & 36,75 & 53,98 & 33,46 & 42,66 & 25,65 & 33,22 \\
\hline Itaquirai & 31,90 & 39,45 & 27,37 & 14,52 & 55,51 & 35,86 & 69,92 & 46,94 & 43,33 & 67,30 & 22,06 & 41,97 & 36,80 & 53,35 & 32,89 & 43,05 & 25,57 & 32,35 \\
\hline Laguna & 33,18 & 39,89 & 26,21 & 13,34 & 55,57 & 36,51 & 70,03 & 47,07 & 42,77 & 67,22 & 22,70 & 42,58 & 37,56 & 53,91 & 33,02 & 43,84 & 25,47 & 31,86 \\
\hline Maracaju & 27,88 & 39,38 & 25,61 & 13,82 & 56,53 & 36,30 & 70,52 & 50,71 & 44,26 & 67,75 & 23,50 & 45,33 & 37,61 & 55,00 & 34,25 & 43,32 & 26,96 & 34,39 \\
\hline Naviraí & 31,86 & 39,30 & 27,06 & 14,40 & 55,46 & 35,79 & 69,88 & 46,82 & 43,19 & 67,25 & 21,97 & 41,91 & 36,79 & 53,31 & 32,75 & 43,03 & 25,34 & 32,26 \\
\hline Nova Alvorada & 27,22 & 38,06 & 25,41 & 13,75 & 55,96 & 34,95 & 70,07 & 48,32 & 43,76 & 67,51 & 21,89 & 43,71 & 36,40 & 53,75 & 33,64 & 42,08 & 26,11 & 33,92 \\
\hline$u_{1}$ & & & & & & & & & & & & & & & & & & \\
\hline Ponta Pora & 31,68 & 40,67 & 26,43 & 13,37 & 56,31 & 37,28 & 70,52 & 49,48 & 43,73 & 68,04 & 23,51 & 44,41 & 38,07 & 54,68 & 33,89 & 44,18 & 26,56 & 32,68 \\
\hline Rio Brilhante & 27,85 & 37,56 & 25,75 & 14,06 & 55,66 & 34,52 & 69,85 & 48,41 & 43,47 & 67,20 & 22,22 & 43,79 & 36,41 & 54,02 & 34,57 & 42,11 & 25,81 & 33,98 \\
\hline Sidrolandia & 23,64 & 40,59 & 25,20 & 14,69 & 58,24 & 37,59 & 71,57 & 54,35 & 46,59 & 69,24 & 23,73 & 47,55 & 38,00 & 55,54 & 35,76 & 43,18 & 29,62 & 36,69 \\
\hline Sonora & 17,58 & 39,55 & 22,92 & 15,64 & 59,61 & 37,36 & 72,18 & 52,81 & 48,46 & 70,21 & 19,29 & 45,50 & 35,45 & 52,82 & 37,51 & 40,66 & 32,74 & 37,73 \\
\hline Max & 36,83 & 40,97 & 27,86 & 15,64 & 59,61 & 38,12 & 72,18 & 54,35 & 48,46 & 70,21 & 23,73 & 47,55 & 38,07 & 55,54 & 37,62 & 44,18 & 32,74 & 40,45 \\
\hline Min & 17,58 & 37,56 & 22,88 & 11,95 & 53,83 & 34,52 & 68,89 & 40,34 & 40,79 & 66,07 & 18,92 & 38,06 & 34,68 & 51,09 & 32,64 & 39,52 & 23,85 & 29,62 \\
\hline Média & 27,87 & 39,22 & 25,54 & 13,88 & 56,46 & 36,14 & 70,45 & 48,60 & 44,23 & 67,91 & 21,84 & 43,61 & 36,72 & 53,66 & 34,25 & 42,66 & 26,95 & 34,24 \\
\hline
\end{tabular}

Legenda: Baixo Alto

MAPE mínimo de $18,92 \%$ em Coxim, máximo de $23,73 \%$ em Sidrolândia e médio de $21,84 \%$. Estes dois métodos apresentaram razoáveis precisões, com $\mathrm{R}^{2}$ aj médio de 0,65 para HAM e 0,75 para MAK (Tabela 6). Contrariando os resultados de MAK encontrados por Reis et al. (2010) e Vescove e Turco (2005).

JH e LIN apresentaram as menores acurácias com MAPE médio de $70,45 \%$ e $70,21 \%$, respectivamente (Tabela 7). LIN apresentou uma baixa precisão média de 0,12 . Outros métodos se mostraram inviáveis para o Estado de Mato Grosso do Sul em escala mensal, como por exemplo, JOB (MAPE médio de $48,60 \%$ e $\mathrm{R}^{2}$ aj médio de 0,07), Tanner-Pelton (TP) com MAPE médio de $42,66 \%$ e $\mathrm{R}^{2}$ aj médio de 0,00 , KHA (MAPE médio de
44,23\% e $\mathrm{R}^{2}$ aj médio de 0,13), BC (MAPE médio de $39,22 \%$ e $\mathrm{R}^{2}$ aj médio de 0,13 ) e BL (MAPE médio de $27,87 \%$ e $\mathrm{R}^{2}$ aj médio de 0.13 ).

Dos métodos que aliaram boa acurácia e precisão para a escala mensal foram MAK e PT que sobressaíram com MAPE médio de $21,84 \%$ e $36,72 \%$ e $\mathrm{R}^{2}$ aj médio de 0,75 e 0,91 , respectivamente (Fig. 5).

A relação entre a evapotranspiração potencial mensal estimada utilizando diferentes modelos e a evapotranspiração potencial mensal por Penman e Monteith (Fig. 5) indicou que os melhores modelos foram HAM e BL. O modelo TP apresentou maior variabilidade e JH foi o modelo que mais superestimou a evapotranspiração potencial mensal no estado de Mato Grosso do Sul. 


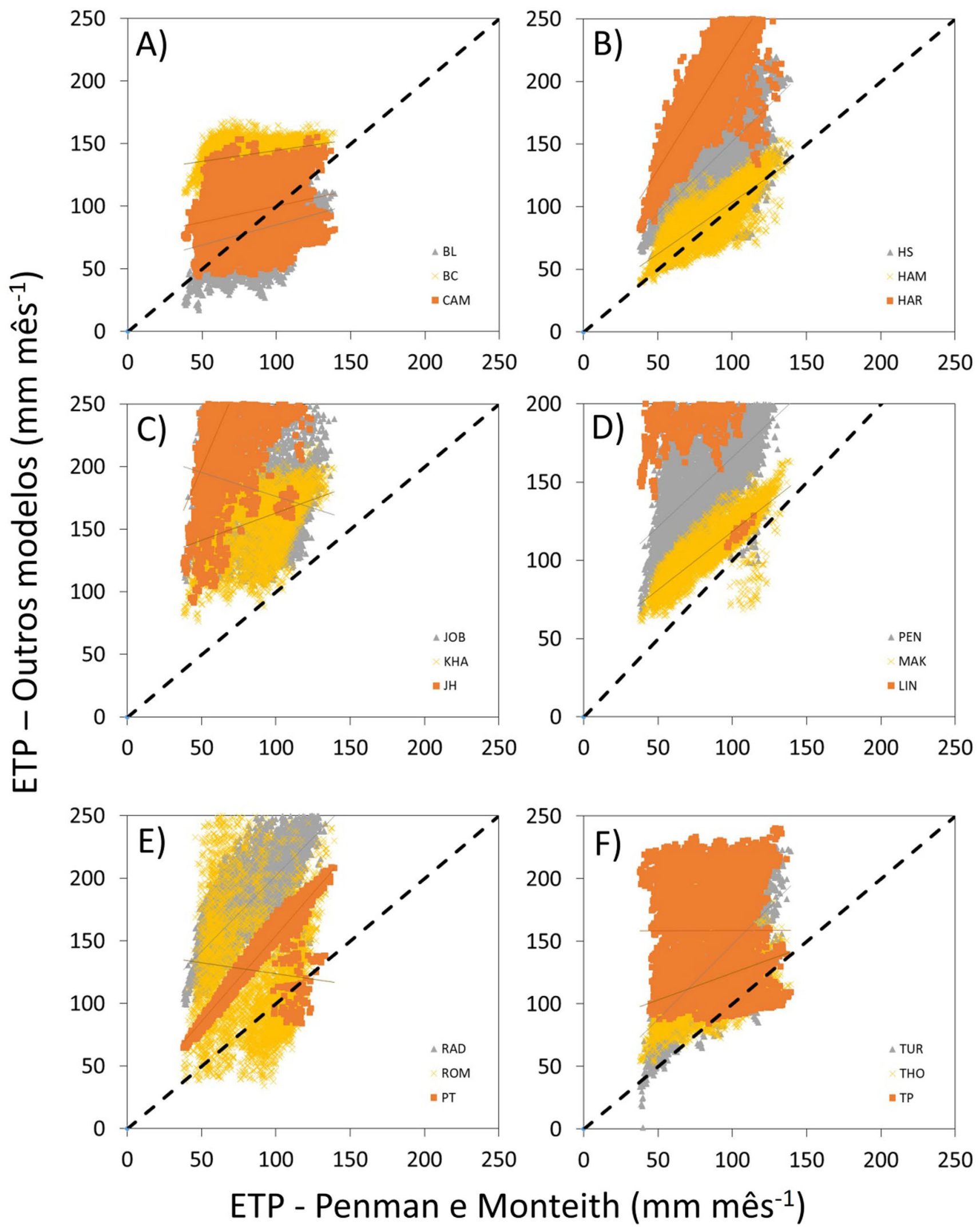

Figura 5 - Desempenho entre os dados Mensal do modelo PM e os modelos (A) Benevidez-Lopez, Blaney-Criddle e Camargo, (B) Hamon, Hargreaves e, Hargreaves-Samani, (C) Jensen-Haise, (H) Jobson, (I) Kharrufa, (D) Linacre, Makkink e, Penman, (E) Priestley-Taylor, Radiation e, Romanenko, (F) Tanner-Pelton, Thornthwaite e Turc. 


\subsection{RMSE e MBE}

Os modelos obtiveram os melhores desempenhos na escala diária (Tabela 8). JH foi o modelo que mais super- estimou nas escalas diária, semanal e mensal. Enquanto BL subestimou em todas as escalas.

Tabela 7 - Desempenho estatístico dos métodos de ETP em escala mensal em relação ao Método Penman-Monteith, considerando a precisão (R2aj). (BC) Benevidez-Lopez, (BL) Blaney-Criddle, (CAM) Camargo, (HAM) Hamon, (HAR) Hargreaves, (HS) Hargreaves-Samani, (JH) Jensen-Haise, (JOB) Jobson, (KHA) Kharrufa, (LIN) Linacre, (MAK) Makkink, (PEN) Penman, (PT) Priestley-Taylor, (RAD) Radiation, (ROM) Romanenko, (TP) Tanner-Pelton, (THO) Thornthwaite e (TUR) Turc.

\begin{tabular}{|c|c|c|c|c|c|c|c|c|c|c|c|c|c|c|c|c|c|c|}
\hline & BL & $\mathrm{BC}$ & CAM & HAM & HAR & HS & JH & JOB & KHA & LIN & MAK & PEN & PT & RAD & ROM & ТP & THO & TUR \\
\hline Amabai & 0,27 & 0,27 & 0,11 & 0,74 & 0,84 & 0,62 & 0,64 & 0,01 & 0,27 & 0,26 & 0,85 & 0,77 & 0,92 & 0,81 & 0,04 & 0,00 & 0,38 & 0,70 \\
\hline Aral Moreira & 0,23 & 0,23 & 0,09 & 0,73 & 0,83 & 0,60 & 0,62 & 0,00 & 0,23 & 0,22 & 0,83 & 0,70 & 0,91 & 0,78 & 0,00 & 0,00 & 0,36 & 0,67 \\
\hline Bandeirantes & 0,02 & 0,02 & 0,02 & 0,65 & 0,81 & 0,55 & 0,54 & 0,19 & 0,02 & 0,02 & 0,73 & 0,32 & 0,90 & 0,60 & 0,16 & 0,00 & 0,10 & 0,62 \\
\hline Caarapo & 0,23 & 0,23 & 0,09 & 0,71 & 0,83 & 0,59 & 0,61 & 0,00 & 0,23 & 0,22 & 0,82 & 0,64 & 0,91 & 0,76 & 0,01 & 0,00 & 0,30 & 0,70 \\
\hline Camapua & 0,03 & 0,03 & 0,02 & 0,58 & 0,78 & 0,47 & 0,47 & 0,17 & 0,03 & 0,02 & 0,65 & 0,21 & 0,89 & 0,48 & 0,13 & 0,00 & 0,10 & 0,56 \\
\hline Campo Grande & 0,04 & 0,04 & 0,02 & 0,62 & 0,81 & 0,51 & 0,51 & 0,18 & 0,04 & 0,03 & 0,69 & 0,22 & 0,90 & 0,52 & 0,13 & 0,00 & 0,03 & 0,62 \\
\hline Chapadao do Sul & 0,02 & 0,02 & 0,02 & 0,55 & 0,74 & 0,45 & 0,44 & 0,12 & 0,02 & 0,01 & 0,64 & 0,21 & 0,89 & 0,47 & 0,09 & 0,00 & 0,20 & 0,50 \\
\hline osta Rica & 0,02 & 0,02 & 0,02 & 0,57 & 0,77 & 0,46 & 0,44 & 0,12 & 0,02 & 0,01 & 0,63 & 0,17 & 0,89 & 0,44 & 0,09 & 0,00 & 0,16 & 0,51 \\
\hline Coxim & 0,00 & 0,00 & 0,00 & 0,49 & 0,76 & 0,42 & 0,41 & 0,16 & 0,00 & 0,00 & 0,57 & 0,12 & 0,90 & 0,37 & 0,13 & 0,00 & 0,08 & 0,55 \\
\hline Douradina & 0,18 & 0,18 & 0,07 & 0,69 & 0,82 & 0,58 & 0,59 & 0,01 & 0,18 & 0,17 & 0,80 & 0,59 & 0,91 & 0,72 & 0,00 & 0,00 & 0,13 & 0,69 \\
\hline Dourados & 0,18 & 0,18 & 0,07 & 0,69 & 0,82 & 0,58 & 0,59 & 0,01 & 0,18 & 0,17 & 0,80 & 0,59 & 0,91 & 0,72 & 0,00 & 0,00 & 0,13 & 0,69 \\
\hline Itaporã & 0,18 & 0,18 & 0,07 & 0,69 & 0,82 & 0,58 & 0,59 & 0,01 & 0,18 & 0,17 & 0,80 & 0,59 & 0,91 & 0,72 & 0,00 & 0,00 & 0,13 & 0,69 \\
\hline Itaquirai & 0,26 & 0,26 & 0,10 & 0,73 & 0,84 & 0,62 & 0,64 & 0,02 & 0,27 & 0,25 & 0,85 & 0,72 & 0,92 & 0,80 & 0,05 & 0,00 & 0,26 & 0,72 \\
\hline Laguna & 0,23 & 0,23 & 0,09 & 0,72 & 0,83 & 0,59 & 0,61 & 0,00 & 0,23 & 0,22 & 0,83 & 0,67 & 0,91 & 0,77 & 0,01 & 0,00 & 0,36 & 0,68 \\
\hline Maracaju & 0,14 & 0,14 & 0,06 & 0,67 & 0,81 & 0,56 & 0,57 & 0,06 & 0,14 & 0,12 & 0,77 & 0,45 & 0,90 & 0,66 & 0,02 & 0,00 & 0,14 & 0,66 \\
\hline Nuvirá & 0,27 & 0,27 & 0,10 & 0,73 & 0,84 & 0,62 & 0,64 & 0,02 & 0,27 & 0,25 & 0,85 & 0,72 & 0,92 & 0,80 & 0,06 & 0,00 & 0,26 & 0,72 \\
\hline Nova Alvorada Sul & 0,11 & 0,11 & 0,04 & 0,65 & 0,81 & 0,55 & 0,55 & 0,07 & 0,11 & 0,10 & 0,77 & 0,48 & 0,91 & 067 & 0,04 & 0,00 & 0,09 & 0,66 \\
\hline Ponta Pora & 0,19 & 0,19 & 0,07 & 0,71 & 0,83 & 0,59 & 0,61 & 0,03 & 0,19 & 0,18 & 0,81 & 0,57 & 0,91 & 0,74 & 0,00 & 0,00 & 0,37 & 0,66 \\
\hline Rio Brilhante & 0,13 & 0,13 & 0,06 & 0,65 & 0,80 & 0,56 & 0,56 & 0,04 & 0,13 & 0,12 & 0,77 & 0,51 & 0,91 & 0,68 & 0,01 & 0,00 & 0,06 & 0,68 \\
\hline Sidrolandia & 0,07 & 0,07 & 0,03 & 0,64 & 0,81 & 0,53 & 0,53 & 0,15 & 0,07 & 0,06 & 0,71 & 0,26 & 0,90 & 0,55 & 0,09 & 0,00 & 0,07 & 0,63 \\
\hline Sonora & 0,00 & 0,00 & 0,00 & 0,45 & 0,74 & 0,39 & 0,37 & 0,13 & 0,00 & 0,00 & 0,53 & 0,08 & 0,90 & 0,33 & 0,10 & 0,00 & 0,05 & 0,51 \\
\hline Max & 0,27 & 0,27 & 0,11 & 0,74 & 0,84 & 0,62 & 0,64 & 0,19 & 0,27 & 0,26 & 0,85 & 0,77 & 0,92 & 0,81 & 0,16 & 0,00 & 0,38 & 0,72 \\
\hline Min & 0,00 & 0,00 & 0,00 & 0,45 & 0,74 & 0,39 & 0,37 & 0,00 & 0,00 & 0,00 & 0,53 & 0,08 & 0,89 & 0,33 & 0,00 & 0,00 & 0,03 & 0,50 \\
\hline Média & 0,13 & 0,13 & 0,05 & 0,65 & 0,81 & 0,54 & 0,55 & 0,07 & 0,13 & 0,12 & 0,75 & 0,46 & 0,91 & 0,64 & 0,06 & 0,00 & 0,18 & 0,64 \\
\hline
\end{tabular}

Legenda: Baixo Alto

Tabela 8 - Desempenho estatístico dos métodos de ETP na escala mensal em relação ao Método Penman-Monteith, considerando a Raiz do Erro Quadrático Médio (RMSE) e o desvio das médias (MBE). (BC) Benevidez-Lopez, (BL) Blaney-Criddle, (CAM) Camargo, (HAM) Hamon, (HAR) Hargreaves, (HS) Hargreaves-Samani, (JH) Jensen-Haise, (JOB) Jobson, (KHA) Kharrufa, (LIN) Linacre, (MAK) Makkink, (PEN) Penman, (PT) PriestleyTaylor, (RAD) Radiation, (ROM) Romanenko, (TP) Tanner-Pelton, (THO) Thornthwaite e (TUR) Turc.

\begin{tabular}{|c|c|c|c|c|c|c|c|c|c|c|c|c|c|c|c|c|c|c|}
\hline PM & BL & $\mathrm{BC}$ & CAM & HAM & HAR & HS & JH & $\mathrm{JOB}$ & KHA & LIN & MAK & PEN & PT & RAD & ROM & TP & THO & TUR \\
\hline \multicolumn{19}{|c|}{ Diário } \\
\hline RMSE & 0.80 & 1.98 & 0.99 & 0.51 & 3.89 & 1.85 & 7.52 & 3.74 & 2.52 & 6.43 & 0.82 & 2.36 & 1.70 & 3.38 & 2.38 & 2.83 & 1.40 & 1.64 \\
\hline MBE & -0.20 & 1.86 & 0.33 & 0.21 & 3.77 & 1.67 & 7.17 & 3.16 & 2.36 & 6.26 & 0.73 & 2.25 & 1.65 & 3.32 & 1.31 & 2.40 & 1.07 & 1.50 \\
\hline \multicolumn{19}{|c|}{ Semanal } \\
\hline RMSE & 18.13 & 20.13 & 16.98 & 17.21 & 29.55 & 19.35 & 52.13 & 32.53 & 22.32 & 45.16 & 17.16 & 21.52 & 19.10 & 26.61 & 22.40 & 22.73 & 18.33 & 19.09 \\
\hline MBE & -4.42 & 9.97 & -0.69 & -1.59 & 23.32 & 8.68 & 47.14 & 24.39 & 13.46 & 40.81 & 2.08 & 12.68 & 8.48 & 20.17 & 6.14 & 13.74 & 4.46 & 7.48 \\
\hline \multicolumn{19}{|c|}{ Mensal } \\
\hline RMSE & 24.01 & 59.46 & 29.80 & 15.19 & 116.70 & 55.58 & 225.64 & 112.05 & 75.67 & 192.89 & 24.50 & 70.82 & 51.01 & 101.48 & 71.54 & 85.00 & 42.15 & 49.34 \\
\hline MBE & -5.92 & 55.73 & 10.04 & 6.22 & 112.97 & 50.20 & 215.06 & 94.68 & 70.71 & 187.91 & 21.94 & 67.37 & 49.37 & 99.45 & 39.34 & 71.91 & 32.15 & 45.09 \\
\hline
\end{tabular}




\section{Conclusões}

Os melhores desempenhos dos modelos de estimativa de evapotranspiração são em escala diária.

Os métodos de Hargreaves e Makkink são mais eficientes para todas as escalas de temporais no Mato Grosso do Sul.

Priestley e Taylor, Hargreaves, Hamon e Makkink apresentam os melhores valores de acurácia e precisão em escala diária para o Estado de Mato Grosso do Sul. Hamon e Makkink para escala semanal, enquanto os melhores métodos para estimar a ETP em escala mensal são Makkink e Priestley e Taylor para o Estado de Mato Grosso do Sul. Apesar de ser simples o uso desses modelos de evapotranspiração potencial nessas escalas temporais, esses precisam apenas dos dados de entrada de temperatura do ar e radiação solar.

\section{Agradecimentos}

Agradecemos ao Instituto Federal de Mato Grosso Sul, Campus Naviraí, pelo financiamento desta pesquisa.

\section{Referências}

ADAMALA, S. Temperature based generalized wavelet-neural network models to estimate evapotranspiration in India. Information processing in agriculture, v. 5, n. 1, p. 149$155,2018$.

ALLEN, R.G.; PEREIRA, L.S.; RAES, D.; SMITH, M. Crop Evapotranspiration-Guidelines for computing crop water requirements-FAO Irrigation and drainage paper 56. Fao, Rome, v. 300, n. 9, p. D05109, 1998. ALMOROX, J.; QUEJ, V.H.; MARTÍ, P. Global performance ranking of temperature-based approaches for evapotranspiration estimation considering Köppen climate classes. Journal of Hydrology, v. 528, p. 514-522, 2015.

ALMOROX, J., et al. Worldwide assessment of the PenmanMonteith temperature approach for the estimation of monthly reference evapotranspiration. Theoretical and applied climatology, v. 131, n. 1-2, p. 693-703, 2018.

BENEVIDES, J.G.; LOPEZ, D. Formula para el caculo de la evapotranspiracion potencial adaptada al tropico $\left(15^{\circ} \mathrm{N}-15^{\circ}\right.$ S). Agronomia Tropical, Maracay, v. 20, n. 5, p. 335-345, 1970.

BLANEY, H.F., et al. Determining water requirements in irrigated areas from climatological and irrigation data. 1950.

BOWIE, G.L.; MILLS, W.B.; PORCELLA, D.B.; CAMPBELL, C.L.; PAGENKOPF, J.R.; RUPP G.L.; JOHNSON, K.M.; CHAN, P.W.H.; GHERINI, S.A.; CHAMBERLIN, C.E. Rates, constants, and kinetics formulations in surface water quality modeling. EPA, v. 600, p. 3-85, 1985.

CAMARGO, A.P. Balanço hídrico no Estado de São Paulo. Campinas: Instituto Agronômico, 24p. Boletim, n. 116, 1971.

CAPORUSSO, N.B.; ROLIM, G. de S. Reference evapotranspiration models using different time scales in the
Jaboticabal region of São Paulo, Brazil. Acta Scientiarum. Agronomy, v. 37, n. 1, p. 1-9, 2015.

CARVALHO, L.G.; RIOS, G.F.A.; MIRANDA, W.L.; CASTRONETO, P. Evapotranspiração de referência: uma abordagem atual de diferentes métodos de estimativa. Pesquisa Agropecuária Tropical, v. 41, n. 3, 2011.

CORNELL, J.A.; BERGER, R.D. Factors that influence the coefficient of determination in single linear and nonlinear models. Phytopathology, Palo Alto, v. 77, p. 63-70, 1987.

CUNHA, F.F.; VENANCIO, L.P.; CAMPOS, F.B.; SEDIYAMA, G.C. Reference evapotranspiration estimates by means of Hargreaves-samani and Penman-monteith fao methods with missing data in the northwestern Mato Grosso do Sul. Bioscience Journal, v. 33, n. 5, p. 1166- 1176, 2017 a.

CUNHA, F.F.D.; MAGALHÃES, F.F.; CASTRO, M.A.D.; SOUZA, E.J.D. Performance of estimative models for daily reference evapotranspiration in the city of Cassilândia, Brazil. Engenharia Agrícola, v. 37, n. 1, p. 173-184, 2017b.

DIDARI, S.; AHMADI, S.H. Calibration and evaluation of the FAO56-Penman-Monteith, FAO24-radiation, and PriestlyTaylor reference evapotranspiration models using the spatially measured solar radiation across a large arid and semiarid area in southern Iran. Theoretical and Applied Climatology, p. 1-15, 2018.

DOORENBOS, J.; PRUITT, W.O. Guidelines for predicting crop water requirements. Food and Agriculture organization. Rome, Irrig. Drainage pap., v. 24, 1977.

FERNANDES, D.S.; HEINEMANN, A.B.; PAZ, R.L.F.; AMORIM, A.O. Evapotranspiração - Uma Revisão sobre os Métodos Empíricos. Santo Antônio de Goiás: Embrapa Arroz e Feijão, 2010. 45p. (Documentos, 263).

GHARBIA, S.S.; SMULLEN, T.; Gill, L.; JOHNSTON, P.; PILLA, F. Spatially distributed potential evapotranspiration modeling and climate projections. Science of the Total Environment, v. 633, p. 571-592, 2018.

HAMON, W.R. Estimating potential evapotranspiration. Journal of Hydraulics Division ASCE, v. 87, n. 3, p. 107-120, 1961.

HARGREAVES, G.H.; ALLEN, R.G. History and evaluation of Hargreaves evapotranspiration equation. Journal of Irrigation and Drainage Engineering, v. 129, n. 1, p. 53-63, 2003.

HARGREAVES, G.L.; SAMANI, Z.A. Reference crop evapotranspiration from temperature. Applied engineering in agriculture, v. 1, n. 2, p. 96-99, 1985.

IRMAK, S.; IRMAK, A.; ALLEN, R.G.; JONES, J.W. Solar and net radiation-based equations to estimate reference evapotranspiration in humid climates. Journal of irrigation and drainage engineering, v. 129, n. 5, p. 336-347, 2003.

JENSEN, M.E.; HAISE, H.R. Estimating evapotranspiration from solar radiation. Journal of the Irrigation and Drainage Division-ASCE, v. 4, n. 1, p. 15-41, 1963.

KHARRUFA, N.S. Simplified equation for evapotranspiration in arid regions. Beitrage zur Hydrologie Sonderheft, v. 5, n. 1, p. 39-47, 1985.

KIAFAR, H.; BABAZADEH, H.; MARTI, P.; KISI, O.; LANDERAS, G.; KARIMI, S.; SHIRI, J. Evaluating the generalizability of GEP models for estimating reference evapotranspiration in distant humid and arid locations. 
Theoretical and Applied Climatology, v. 130, n. 1-2, p. 377-389, 2017.

LINACRE, E. T. A. Simple formula for estimating evaporation rates in various climates, using temperature data alone. Agricultural Meteorology, v. 18, n.1, p. 409-424, 1977.

MA, F.; LIU, D.; FU, Q.; WRZESINSKI, D.; BAIG, F.; NABI, G.; MI, K.; LI, T.; CUI, S. Extreme precipitation and drought monitoring in northeastern China using general circulation models and pan evaporation-based drought indices. Climate Research, v. 74, n. 3, p. 231-250, 2018.

MAČEK, U.; BEZAK, N.; ŠRAJ, M. Reference evapotranspiration changes in Slovenia, Europe. Agricultural and forest meteorology, v. 260, p. 183-192, 2018.

MAKKINK, G. F. Testing the Penman formula by means of lysimeters. Journal of the Institution of Water Engineerrs, v. 11, p. 277-288, 1957.

MCCABE, G.J.; HAY, L.E.; BOCK, A.; MARKSTROM, S.L.; ATKINSON, R.D. Inter-annual and spatial variability of Hamon potential evapotranspiration model coefficients. Journal of Hydrology, v. 521, p. 389-394, 2015.

MEDINA, H.; TIAN, D.; SRIVASTAVA, P.; PELOSI, A.; CHIRICO, G.B. Medium-range reference evapotranspiration forecasts for the contiguous United States based on multimodel numerical weather predictions. Journal of hydrology, v. 562, p. 502-517, 2018. MEHDIZADEH, S.; SAADATNEJADGHARAHASSANLOU, H.; BEHMANESH, J. Calibration of Hargreaves-Samani and Priestley-Taylor equations in estimating reference evapotranspiration in the Northwest of Iran. Archives of Agronomy and Soil Science, v. 63, n. 7, p. 942-955, 2017.

MOKHTARI, A.; NOORY, H.; VAZIFEDOUST, M.; BAHRAMI, M. Estimating net irrigation requirement of winter wheat using model-and satellite-based single and basal crop coefficients. Agricultural water management, v. 208, p. 95-106, 2018.

MORAES, S.; LOPES, S.; FANAYA, E.D.; MARINHO, V.G.; PEREIRA, F.; DEISIANE, C.; COSTA, O.; CHERRI, U. Comparative performance of empirical methods to estimate the reference evapotranspiration in Aquidauana, MS, Brazil. Científica, v. 46, n. 2, p. 143-150, 2018.

MOELETSI, Mokhele Edmond; WALKER, Sue. Assessment of agricultural drought using a simple water balance model in the Free State Province of South Africa. Theoretical and applied climatology, v. 108, n. 3-4, p. 425-450, 2012.

NASA-POWER. Prediction Of Worldwide Energy Resource. 2019. Disponível em: <https://power.larc.nasa.gov/com mon/php/POWER_AboutAgroclimatology.php $>$. Acesso em: 13 mar. 2019.

PENG, L.; LI, Y.; FENG, H. The best alternative for estimating reference crop evapotranspiration in different sub-regions of mainland China. Scientific reports, v. 7, n. 1, p. 5458 , 2017.

PENMAN, H.L. Natural evaporation from open water, bare soil and grass. Proceedings of the Royal Society of London. Series A. Mathematical and Physical Sciences, v. 193, n. 1032, p. 120-145, 1948.
PRIESTLEY, C.H.B.; TAYLOR, R.J. On the assessment of surface heat flux and evaporation using large-scale parameters. Monthly weather review, v. 100, n. 2, p. 81-92, 1972.

RAMAROHETRA, J.; SULTAN, B. Impact of ET0 method on the simulation of historical and future crop yields: a case study of millet growth in Senegal. International Journal of Climatology, v. 38, n. 2, p. 729-741, 2018.

REIS, E.F. de; BRAGANÇA, R. de; GARCIA, G.O. Estudo comparativo da estimativa da evapotranspiração de referência no período chuvoso para três localidades no estado do Espírito Santo. Idesia (Arica), v. 28, n. 2, p. 21-29, 2010.

ROMANENKO, V.A. Computation of the autumn soil moisture using a universal relationship for a large area. Proc. of Ukrainian Hydrometeorological Research Institute, v. 3, p. 12-25, 1961.

SANTOS, A.A.R.; LYRA, G.B.; LYRA, G.B.; LIMA, E.P.; SOUZA, J.L. de. Delgado, R.C. Estimates of the reference evapotranspiration using air temperature extremes in the state of the Rio de Janeiro, southeastern Brazil. IRRIGA, v. 21, n. 3, p. 449-465, 2016.

SANTOS, D.P.; SANTOS, C.S.; SILVA, L.M.; SANTOS, M.A.L. ; SANTOS, C.G. Performance of methods for estimation of table beet water requirement in Alagoas. Revista Brasileira de Engenharia Agrícola e Ambiental, v. 22, n. 3, p. 189-193, 2018.

SEONG, C.; SRIDHAR, V.; BILLAH, M.M. Implications of potential evapotranspiration methods for streamflow estimations under changing climatic conditions. International Journal of Climatology, v. 38, n. 2, p. 896-914, 2018.

TABARI, H.; GRISMER, M.E.; TRAJKOVIC, S. Comparative analysis of 31 reference evapotranspiration methods under humid conditions. Irrigation Science, v. 31, n. 2, p. 107117, 2013.

TANAKA, A.A.; SOUZA, A.P. de.; KLAR, A.E., SILVA, A.C. da.; GOMES, A.W.A. Evapotranspiração de referência estimada por modelos simplificados para o Estado do Mato Grosso. Pesquisa Agropecuária Brasileira, v. 51, n. 2, p. 91-104, 2016.

TANNER, C.O.; PELTON, W.L. Potential evapotranspiration estimates by the approximate energy balance method of Penman. Journal of geophysical research, v. 65 , n. 10 , p. 3391-3413, 1960.

TAO, H.; DIOP, L.; BODIAN, A.; DJAMAN, K.; NDIAYE, P.M.; YASEEN, Z.M. Reference evapotranspiration prediction using hybridized fuzzy model with firefly algorithm: Regional case study in Burkina Faso. Agricultural water management, v. 208, p. 140-151, 2018.

THORNTHWAITE, C. W. An approach toward a rational classification of climate. Geographical Review, v. 38, n. 1, p. 55-94, 1948.

TOMAS-BURGUERA, M.; VICENTE-SERRANO, S.M.; GRIMALT, M.; BEGUERÍA, S. Accuracy of reference evapotranspiration (ETo) estimates under data scarcity scenarios in the Iberian Peninsula. Agricultural water management, v. 182, p. 103-116, 2017. 
TRAJKOVIC, S.; KOLAKOVIC, S. Evaluation of reference evapotranspiration equations under humid conditions. Water Resource Management, Amsterdam, v. 23, n. 14, p. 3057-3067, 2009.

TRIGO, I.F.; BRUIN, H. de; BEYRICH, F.; BOSVELD, F.C.; GAVILÁN, P.; GROH, J.; LÓPEZ-URREA, R. Validation of reference evapotranspiration from Meteosat Second Generation (MSG) observations. Agricultural and forest meteorology, v. 259, p. 271-285, 2018.

TURC, L. Estimation of irrigation water requirements, potential evapotranspiration: a simple climatic formula evolved up to date. Ann. Agron, v. 12, n. 1, p. 13-49, 1961.
VALIANTZAS, J. D. Temperature-and humidity-based simplified Penman's ET0 formulae. Comparisons with temperature-based Hargreaves-Samani and other methodologies. Agricultural water management, v. 208, p. 326-334, 2018.

VESCOVE, H.V.; TURCO, J.E.P. Comparação de três métodos de estimativa da evapotranspiração de referência para a região de Araraquara-SP. Engenharia Agrícola, p. 713721, 2005.

License information: This is an open-access article distributed under the terms of the Creative Commons Attribution License (type CC-BY), which permits unrestricted use, distribution and reproduction in any medium, provided the original article is properly cited. 